\title{
Does entropy modulate the prediction of German long-distance verb particles?
}

Kate Stone ${ }^{* 1 \odot}$, Shravan Vasishth ${ }^{1 \odot}$, Titus von der Malsburg ${ }^{2 \not \subset}$

1 Department of Linguistics, University of Potsdam, Germany

2 Institute of Linguistics, University of Stuttgart, Germany

(C) Current Address: Department of Linguistics, University of Potsdam, Karl-Liebknecht-Straße 24-25, 14476 Potsdam, Germany

¿Current Address: Institute of Linguistics, University of Stuttgart, Keplerstraße 17, 70174, Germany

* Corresponding author

E-mail: stone@uni-potsdam.de

\section{Abstract}

In this paper we examine the effect of uncertainty on readers' predictions about meaning. In particular, we were interested in how uncertainty might influence the likelihood of committing to a specific sentence meaning. We conducted two event-related potential (ERP) experiments using particle verbs such as turn down and manipulated uncertainty by constraining the context such that readers could be either highly certain about the identity of a distant verb particle, such as turn the bed [...] down, or less certain due to competing particles, such as turn the music [...] up/down. The study was conducted in German, where verb particles appear clause-finally and may be separated from the verb by a large amount of material. We hypothesised that this separation would encourage 
readers to predict the particle, and that high certainty would make prediction of a specific particle more likely than lower certainty. If a specific particle was predicted, this would reflect a strong commitment to sentence meaning that should incur a higher processing cost if the prediction is wrong. If a specific particle was less likely to be predicted, commitment should be weaker and the processing cost of a wrong prediction lower. If true, this could suggest that uncertainty discourages predictions via an unacceptable cost-benefit ratio. However, given the clear predictions made by the literature, it was surprisingly unclear whether the uncertainty manipulation affected the two ERP components studied, the N400 and the PNP. Bayes factor analyses showed that evidence for small a priori hypothesised effect sizes was inconclusive, although there was decisive evidence against large a priori hypothesised effect sizes greater than $1 \mu V$ for the N400 and greater than $3 \mu V$ for the PNP. We attribute the inconclusive finding to the properties of verb-particle dependencies that differ from the verb-noun dependencies in which the N400 and PNP are often studied.

\section{Introduction}

Contextual cues in a sentence preactivate words in memory, such that processing $\quad 2$ of those words begins before they are seen [1,2]. If contextual cues are 3 sufficiently constraining, preactivation may crystallise into prediction of a 4 specific word. Such lexical predictions can benefit readers in processing 5 long-distance dependencies, where key information to interpreting an event is 6 delayed [3 5]. Take for example the German particle verb construction in (1), 7 where a large amount of material separates the verb "fuhr" carried from its 8 particle "fort" on:

Der Professor fuhr mit seinem Vortrag trotz regelmäßiger $\quad 10$ The professor carried with his lecture despite regular Störungen fort. interruptions on. 
Rather than waiting for the particle to appear, and thus waiting to know the 12 exact meaning of the sentence, readers may predict "fort" in order to facilitate 13 processing of the intervening material. However, other plausible particles could 14 include "ab" (the professor drove off), "zusammen" (the professor was startled), 15 or "zurück" (the professor reversed [his position] or drove back). This creates 16 uncertainty about the intended meaning of the sentence. In this paper, we use 17 German particle verbs to test how uncertainty may affect predictive processing 18 difficulty in long-distance dependencies. Although we did not find a conclusive 19 answer to this question, we report our attempt as a resource for future 20 researchers who may wish to revisit the issue. Furthermore, by reporting the $\quad{ }_{21}$ inconclusive results, we avoid contributing to publication bias via the file drawer ${ }_{22}$ problem [6].

\section{Prediction in long-distance dependencies}

Evidence that readers make long-distance predictions is most often observed for $\quad 25$ syntactic information, including in verb-particle constructions. Piai et al. (2013) 26 found evidence that Dutch verbs that could take a particle elicited a larger left $\quad 27$ anterior negativity (LAN) than those that never took a particle. The LAN has ${ }_{28}$ been associated with maintaining information in working memory [7], 29 suggesting that the verb triggered a prediction for a particle. However, there 30 was no evidence that the number of different particles licensed by the verb 31 affected LAN amplitude, suggesting that the prediction was for the syntactic 32 features of the verb-particle dependency and not for its lexical properties. The 33 LAN effect was still present at the direct object, suggesting that readers $\quad 34$ maintained their syntactic prediction while processing the intervening sentence. 35

Further evidence for long-distance syntactic predictions comes from filler-gap 36 dependencies such as I know what $t_{i}$ you hit and broke the mirror with _ $i$ [8]. 37 Encountering a transitive verb within a $w h$-dependency is argued to induce $\quad 38$ active prediction of a gap site, inducing longer reading times in the region of hit 39 
than at the same point in the non-wh-dependency sentence $I$ know that you hit ${ }_{40}$ and broke the mirror with the ball [8-11]. This predicted gap is assumed to be ${ }_{41}$ maintained until it is resolved. Evidence from event-related potentials (ERPs) ${ }_{42}$ supports predicted gap maintenance by showing that a sustained anterior $\quad 43$ negativity (SAN) is larger for wh-questions that are expected to be long vs. $\quad{ }_{44}$ short; this has been attributed to a higher burden placed on working memory $\quad 45$ when a longer structure is expected $[12[14] . \quad 46$

Maintenance of a syntactic prediction should facilitate downstream $\quad 47$ processing, and indeed such facilitation has been observed in reading times. For ${ }_{48}$ example, encountering the word either facilitates processing of a co-ordinate or ${ }_{49}$ structure in sentences like "The team took either the train or the subway..." 50 compared to "The team took the train or the subway..." 15, 16. This suggests 51 that either triggers a syntactic prediction for the co-ordinate structure, which is $\quad 52$ sustained to facilitate reading at its resolution. Staub and colleagues also 53 observed that syntactic predictions facilitated processing by protecting against $\quad 54$ misanalysis of S-coordination as NP-coordination. When either was present in $\quad 55$ sentences such as "Either Linda bought the red car or her husband leased the $\quad 56$ green one", the NP her husband was never misanalysed as the direct object of $\quad 57$ Linda bought. However, when either was absent, a strong preference for 58 NP-coordination appeared to trigger misanalysis, as suggested by a larger $\quad 59$ number of regressions in the spillover region following her husband. 60 Long-distance syntactic predictions may therefore facilitate processing through $\quad 61$ both preactivation and protection against misanalysis. 62

In contrast to syntactic prediction, lexical prediction may represent 63 commitment to a more specific sentence interpretation. Specific predictions can $\quad 64$ increase the facilitatory advantage of a correct prediction, but also run an $\quad 65$ increased risk of costly misprediction. The increased risk may explain why Piai ${ }_{66}$ and colleagues (2013) did not find ERP evidence of lexical prediction in their $\quad 67$ $\begin{array}{ll}\text { particle verb stimuli, as discussed above. } & 68\end{array}$ 
Commitment to a specific sentence interpretation refers to one account of the $\quad 69$ $\begin{array}{ll}\text { predictive mechanism in sentence processing where a specific interpretation } & 70\end{array}$ might be stored in working memory $1,2,17,18$. However, the mechanisms 71 underlying predictive processing are by no means resolved. An alternative 72 account is that the level of preactivation alone is sufficient to explain 73 predictability effects [19]. This is supported by the success of metrics such as 74 surprisal that measure the probability of a word given its context — which could 75 be assumed to be linked to word preactivation - in accounting for variability in 76 predictability-related empirical effects $[20-25]$. On the other hand, it has been 77 shown that suprisal alone is not sufficient to explain all differences in reading $\quad 78$ times or the N400 26, 27]. In the case of reading times, this was taken to 79 suggest the need of an additional explanatory factors such as reanalysis or $\quad 80$ revision [26]. Assuming that reanalysis or revision can only be triggered if a $\quad{ }_{81}$ commitment has been made, such findings could be taken as evidence that $\quad 82$ predictions can involve commitments. 83

On the other hand, reading time studies do provide some support for $\quad 84$ long-distance lexical predictions. A study of particle verb-like constructions in $\quad 85$ Persian found that increased lexical predictability of a head-final verb facilitated $\quad 86$ reading times in both short- and long-distance dependencies [4]. One 87 interpretation of these results is that readers predicted the verb early in the $\quad 88$ sentence and sustained this prediction until it was resolved at the verb site. $\quad 89$ However, the pre-verbal information in both short- and long-distance conditions $\quad 90$ may have been informative about the identity of the verb, and so it is possible $\quad 91$ that the verb in this study was only predicted immediately prior to its being $\quad 92$ $\begin{array}{ll}\text { seen. } & 93\end{array}$

In similar constructions in Hindi, increased lexical predictability was also $\quad 94$ found to facilitate reading times at both short and long distance 3 . The 95 pre-verbal region in this study was not informative about the identity of the $\quad 96$ verb, and so it could reasonably be concluded that the predictability effect in $\quad 97$ 
the long-distance condition was evidence of long-distance lexical predictions. $\quad 98$

In support of the Hindi findings, a study of German particle verbs found that $\quad 99$ higher certainty about the identity of a particle facilitated eye fixation times at 100 the particle in both short- and long-distance conditions [5. In this study again, 101 the pre-particle region was not informative about the identity of the particle, $\quad{ }_{102}$ supporting the conclusion that long-distance predictions may have been made. ${ }_{103}$ However, the uncertainty effect was not replicated in self-paced reading times, 104 suggesting that the effect of lexical certainty on eye movements may have been 105 $\begin{array}{ll}\text { quite subtle. } & 106\end{array}$

Taken together, the above studies suggest that long-distance predictions are 107 generated and maintained to facilitate the processing of long-distance $\quad 108$ dependencies. However, whether lexical content is included in these predictions 109 appears to be more difficult to observe. One possibility is that higher variability 110 among lexical versus syntactic continuations of a sentence creates a higher $\quad 111$ degree of uncertainty for the parser. Combined with the likelihood that 112 intervening material may require the long-distance lexical prediction to be $\quad 113$ reanalysed, lexical predictions may be discouraged in all but the highest $\quad 114$ certainty situations. Even the potential facilitatory benefit of a correct 115 prediction to processing the predicted word and the intervening material may be 116 $\begin{array}{ll}\text { outweighed by potential reanalysis cost. } & 117\end{array}$

\section{The cost of uncertainty in predictive processing 118}

The probability of a misprediction increases as uncertainty about a sentence $\quad{ }_{119}$ increases, making uncertainty a potentially important factor in whether readers 120 make predictions about long-distance dependencies. A reader's uncertainty at a ${ }_{121}$ given point in a sentence can be measured with entropy, which quantifies the $\quad{ }_{122}$ distribution of different ways a sentence could continue [20]; for example, via ${ }_{123}$ the number of different continuations provided at a particular point in the $\quad{ }_{124}$ sentence in a sentence-completion task. A link between entropy and predictive ${ }_{125}$ 
processing cost has been observed in reading times. Encountering a word that 126 triggers entropy among plausible sentence continuations has been associated $\quad{ }_{127}$ with longer reading times, suggesting that uncertainty creates processing cost $\quad{ }_{128}$ via the prediction and maintenance of multiple potential continuations in $\quad 129$ memory 28, 29]. Large reductions in entropy have also been associated with $\quad 130$ increased reading times, suggesting that large shifts in expectation also incur a ${ }_{131}$ processing cost $20,24,30,31$. 3132

The relationship between entropy and processing cost is less well-studied in 133 event-related potentials (ERP). Several experiments have examined the effect of 134 contextual constraint on predictive processing, and constraint can be interpreted 135 as a measure of entropy at a target region of a sentence. However, constraint in 136 such studies is usually quantified using the highest probability word given at the 137 target region in a sentence-completion task (a cloze test); for example, see $\quad 138$ 32 34]. The degree of entropy among different cloze test completions is usually 139 not taken into account. 140

Entropy among cloze test completions is important if we assume that it $\quad{ }_{141}$ represents memory activations in a single reader [35]. Consider a sentence 142 where the most strongly activated word at a given point has a cloze probability ${ }_{143}$ of $36 \%$ and that two other activated words each have a probability of $32 \%$ (high $\quad 144$ entropy). A reader would be highly uncertain about how the sentence will $\quad 145$ continue, given there are three almost equally probable options. Compare this to ${ }_{146}$ a sentence where the strongest activated word also has a probability of $36 \%$, but ${ }_{147}$ where there is a large spread of other activated words each with a probability of 148 no more than $5 \%$ (lower entropy). The reader can now be relatively certain $\quad{ }_{149}$ about how the sentence will continue, even though the target word has the same $\quad 150$ probability as in the first sentence. Under the highest-cloze-probability 151 definition of constraint used in many ERP studies, both sentences would be $\quad{ }^{152}$ grouped together as weakly constraining, even though the difference in 153 uncertainty will have different consequences for predictive processing. $\quad 154$ 
The highest-cloze-probability definition of constraint may explain 155 inconsistency among ERP findings about the effect of constraint on processing 156 cost. In particular, studies have observed a larger anterior late positivity (PNP) 157 for unexpected words in strongly versus weakly constraining contexts, suggesting 158 that higher certainty leads to predictions which are more costly when $\quad 159$ disconfirmed $32,33,36$. The PNP has been linked to the suppression of $\quad 160$ disconfirmed sentence representations, potentially shedding light on the $\quad 161$ mechanism driving the processing costs observed in reading times [37. 162 However, not all studies of the PNP find an effect of constraint, or have $\quad 163$ observed it in the opposite direction $34,38,42$. 164

Few studies have directly tested entropy in ERP, as opposed to constraint. In 165 an exploratory analysis of Thornhill and Van Petten's (2012) data, a reduction 166 in entropy at the target word was found to explain some variance in the PNP, $\quad{ }_{167}$ although this did not survive a confirmatory analysis [23]. In Piai et al.'s (2013) 168 study of Dutch particle verbs, verbs that took large number of particles were not ${ }_{169}$ found to elicit differences in the left anterior negativity (LAN) compared to $\quad 170$ verbs that took a small number of particles. While entropy among particles was 171 not explicitly quantified, the findings can be interpreted as showing that $\quad 172$ differences in lexical uncertainty did not result in differences in working memory 173 burden, as indexed by the LAN. The processing cost of disconfirmed predictions 174 in relation to entropy was not investigated. Given that uncertainty may be a $\quad{ }^{175}$ deciding factor in predictive processing of long-distance dependencies and that 176 ERP has the potential to reveal mechanisms driving processing cost, we $\quad 177$ conducted two ERP experiments examining the effect of entropy on predictive 178 $\begin{array}{ll}\text { processing. } & 179\end{array}$

\section{$\begin{array}{ll}\text { The current experiments } & 180\end{array}$}

The aim of the experiments was to test whether lexical entropy would affect $\quad 181$ readers' willingness to predict a long-distance verb particle. We compared 182 
sentences which required a particle to be semantically plausible, but varied in $\quad{ }^{183}$ how many different particles could complete the sentence. Since we were $\quad 184$ specifically interested in the effect of entropy among the different particles' 185 lexical representations, it was strictly controlled: In the example experimental 186 item (2) below, only a single particle was semantically plausible in conditions (a) 187 and (b). In conditions (c) and (d), at least two particles were plausible. We $\quad 188$ hypothesised that readers should experience more uncertainty about the $\quad 189$ meaning of the sentence in (c/d), and thus be less likely to predict a specific $\quad 190$ particle given the increased potential for a misprediction and its associated cost: 191

(2) 1 plausible particle, expected:

192

a. Der ordentliche Professor fuhr mit seinem Vortrag trotz 193 The orderly professor carried with his lecture despite regelmäßiger Störungen immer ordnungsgemäß fort, da er für 194 regular interruptions always properly on, as he for seine Unaufgeregtheit bekannt war. his unflappability known was.

1 plausible particle, violation: $\quad 196$

b. *Der ordentliche Professor fuhr mit seinem Vortrag trotz 197 The orderly professor carried with his lecture despite regelmäßiger Störungen immer ordnungsgemäß mit, da er für 198 regular interruptions always properly with, as he for seine Unaufgeregtheit bekannt war. his unflappability known was.

$2+$ plausible particles, expected:

c. Der ordentliche Buchhalter fuhr seinen zuverlässigen 201 The orderly accountant turned his reliable Computer bei der Arbeit immer ordnungsgemäß herunter/hoch, 202 computer at work always properly off/on,

da er für seine korrekte Arbeitsweise bekannt war. as he for his correct work practices known was.

$2+$ plausible particles, violation: 204

d. *Der ordentliche Buchhalter fuhr seinen zuverlässigen 205 The orderly accountant turned his reliable 
Computer bei der Arbeit immer ordnungsgemäß mit, da er für computer at work always properly with, as he for seine korrekte Arbeitsweise bekannt war. 207 his correct work practices known was.

We used the N400 and the post-anterior positivity (PNP) to measure 208 processing cost at the particle. In particular, we were interested in ERP 209 evidence of processing cost when an unexpected, semantically implausible $\quad 210$ particle was encountered in conditions (b) and (d). We focused our inference on ${ }^{211}$ these conditions because the target particles both had a cloze probability of zero. ${ }_{212}$ This allowed us to rule out the possibility that any ERP difference observed at ${ }_{213}$ the particle was due to differences in cloze probability, which correlate strongly 214 with amplitude of the N400 and the PNP 33, 43. While cloze probability is a 215 measure of a word's predictability, it does not necessarily equate to whether a ${ }_{216}$ word is predicted: Preactivation of a word by its context - which increases cloze ${ }_{217}$ probability 35 -also makes that word easier to access in the lexicon and $\quad 218$ integrate into the building sentence representation once it is seen, without it $\quad{ }_{219}$ having been specifically predicted 1 Differences in ERP amplitude associated $\quad 220$ with a target word's cloze probability may therefore also be driven by $\quad 221$ non-predictive processes triggered by having seen that word. In contrast, a 222 difference between two target words with the same cloze probability but 223 differing in entropy - the distribution of words not seen but 224 preactivated - should be informative about predictive processing. The target ${ }_{225}$ words in (b) and (d) were always identical, and the pre- and post-critical regions 226 were matched between conditions to rule out any influence of the preceding or $\quad{ }_{227}$ following words on the ERP. The pre-critical region was not informative about $\quad 228$ the identity of the particle. 229

We use the term "zero probability" when referring to the implausible 230 particles to reflect the fact that the sentence context is highly unlikely to 231

\footnotetext{
${ }^{1}$ We assume a difference between preactivation and prediction, where preactivation can reflect activation of several different items in memory, while prediction reflects commitment to one specific item, for example by adding it to working memory $1,2,17,18$.
} 
preactivate implausible particles in memory as they would be meaningless in the ${ }_{232}$ context. However, there exists evidence from the N400 demonstrating that 233 readers can be sensitive to differences within the very low end of the probability ${ }_{234}$ spectrum, including for implausible words 25. These activations are unlikely ${ }_{235}$ to be captured by a cloze test and thus have a cloze probability of zero, but $\quad 236$ their preactivation may still affect ERP amplitude. This would be a caveat to $\quad{ }_{237}$ our "matched zero probability" target particles. The contribution of very low 238 probability words to entropy and ERP amplitude needs future investigation, but ${ }_{239}$ for the current study we use the term "zero probability" to refer broadly to a 240 situation where, for the purposes of studying the strength of people's 241 probabilistic representations of the context of a sentence, the contribution of $\quad{ }_{242}$ preactivation from an implausible particle is effectively zero. 243

To detect the cost of violated predictions, we examined two ERP 244 components: the N400 and the anterior post-N400 positivity (PNP). The N400 245 is a negative deflection in the ERP occurring at around 250-500 ms after a word 246 is seen and is highly correlated with a word's probability given the preceding $\quad 247$ sentence context [43]. Amplitude of the N400 decreases at each new, 248 context-congruent word in a sentence [44,45] and increases at any unexpected 249 word, inversely proportional to its probability 43, 46]. This has led to the 250 hypothesis that sentence context allows the preactivation of probable words that 251 are then easier to process once encountered, and that the N400 reflects the $\quad{ }_{252}$ lexical access or update cost incurred by words with low preactivation [43, 47]. 253

Previous studies involving uncertainty have tested the effect of contextual $\quad 254$ constraint on the N400, but have not found that constraint affects its amplitude 255 when the cloze probability of the target words is matched $[32,33,41,46,48$; but 256 see 49,50 . That is, two words with the same low probability will trigger N400s ${ }_{257}$ of the same amplitude, even if one of them is found in a strongly constraining $\quad 258$ context and the other in a weakly constraining context. Lexical access and $\quad 259$ probabilistic update models of the N400 explain this as occurring because each $\quad 260$ 
low probability word has received no preactivation, meaning that lexical access is 261 equally unfacilitated [43 or that the shift in model update is equally large [47]. 262 This presents a testable null hypothesis for the current study: if the N400 is not 263 affected by constraint - which should reflect entropy - then we should not expect 264 to see a difference in N400 amplitude between 1- and 2+particle violations. $\quad 265$ However, given that few previous studies of the N400 have directly tested 266 entropy, it is possible that we could see a larger N400 in the 1-particle condition 267 reflecting greater update or more difficult lexical access after a misprediction. $\quad 268$

The PNP is a positive amplitude deflection in the anterior region of the scalp ${ }_{269}$ in the 600-900 ms window $32,33,46$. The PNP appears to be functionally 270 distinct from the more well-known posterior P600 [32, 33], which has been $\quad 271$ associated with conflict detection and repair processes [51 [56]. In contrast to ${ }_{272}$ the P600, the PNP has been associated with suppression of disconfirmed 273 sentence representations 33,37 and appears only to be sensitive to plausible $\quad 274$ but unexpected words, suggesting that suppression may only occur if there is a 275 viable, alternative way to update the event representation [32, 33, 57]. If this is 276 the case, we may not expect to see a PNP modulation by our implausible $\quad 277$ $\begin{array}{ll}\text { particles. } & 278\end{array}$

However, in addition to inconsistent findings about the effect of constraint on 279 the PNP already mentioned $34,38,42$, the plausibility feature has only ever 280 been tested in verb-noun dependencies. A context-violating noun may be 281 implausible at multiple levels of representation (e.g. animacy, thematic role, ${ }_{282}$ semantic), whereas our implausible particles present a purely semantic violation. 283 It is thus less clear how the PNP might be affected. If purely semantic violations ${ }_{284}$ can elicit the PNP, we may see a higher cost (larger PNP) of having to suppress 285 a disconfirmed sentence representation at the violation particle in the 1-particle 286 condition than in the $2+$ particle condition. If not, then we may see no difference 287 associated with entropy if the PNP is not triggered by the implausible violations. 288

We tested these hypotheses in two ERP experiments: in Experiment 1, we ${ }_{289}$ 
used the $2 \times 2$ design set out in example (2) to compare the effect of the $\quad 290$

implausible particles on the N400 and PNP, but also to confirm that the 291

particles could elicit an N400 and PNP in the implausible versus plausible $\quad 292$

conditions. In Experiment 2, we attempted to replicate the findings of 293

Experiment 1 using a simpler design with a larger number of participants and $\quad 294$

experimental items. Data, code, and materials for both experiments can be 295

found at https://osf.io/h75jm/. 296

$\begin{array}{ll}\text { Experiment } 1 & \\ & \end{array}$

Experiment 1 tested whether entropy would affect readers' predictions about an ${ }_{298}$ upcoming verb particle. We inferred that predictions had been made by 299 measuring processing cost at prediction violations. We predicted that a larger 300 N400 and PNP would reflect the greater cost of recovering from a mis-prediction 301 in the 1-particle condition than in the $2+$ particle condition where a prediction $\quad 302$ was less likely. Experiment 1 was pre-registered at https://osf.io/qbna2; see 303 \begin{tabular}{ll} 
also S1 Appendix & 304 \\
\hline
\end{tabular}

$\begin{array}{ll}\text { Materials and methods } & 305\end{array}$

$\begin{array}{ll}\text { Participants } & 306\end{array}$

Fifty-four participants were recruited. The sample size was determined by 307 recruiting as many participants as was possible during one university semester. $\quad 308$ Four participants were excluded due to a large number of their target EEG $\quad 309$ trials being contaminated by muscle and/or blink artefacts. This left a total of 310 50 subjects (6 male), with a mean age of 25 years (range: $17-40$ years, $S D=5 \quad 311$ years). In line with university policy, all participants were reimbursed for their 312 time either financially or in the form of credit points toward their studies. All 313 participants were right-handed German native speakers, with no known history 314 of developmental or current language, neurological, or psychiatric disorder, and 315 
had not participated in the cloze test. The study was conducted in line with the 316 principles of the Declaration of Helsinki and all participants provided written $\quad 317$ consent to participation in the study. In accordance with German law, IRB 318 review was not required as the study involved only healthy adult participants. $\quad 319$

\section{Materials}

For each particle verb, two sentences were constructed, as seen in Example (2). 321 The position of the base verb and particle in each sentence pair was matched. $\quad 322$ The particle was the target word. Within each sentence pair, at least two words 323 before the particle were identical. 103 sentence pairs were constructed and $\quad 324$ presented as a cloze test to 30 German native speakers (mean age 25 years, SD $6 \quad 325$ years, range 18-41 years) on a desktop computer in our in-house lab using the $\quad 326$ Ibex software [58]. The sentence pairs were divided into two lists, such that 327 each participant only saw one condition from each item. The particle of the 328 sentence was replaced by a gap, which participants were asked to fill with the $\quad{ }_{329}$ first word that came to mind.

The items were then ranked in terms of how well they fulfilled the criteria 331 that the 1-particle condition elicited only one particle and the $2+$ particle 332 condition elicited at least two particles with similar probability. To rank the 333 items, cloze probabilities were calculated for each particle completion. Other $\quad 334$ kinds of completions were grouped into categories (e.g. prepositional phrases, $\quad 335$ adjectives, nouns) and a cloze probability was calculated for each category. $\quad 336$ Items were then ranked by entropy among the responses (lowest to highest in $\quad 337$ the 1-particle condition; highest to lowest in the 2+particle condition). For the 338 $2+$ particle condition, further weight was given to items where the two highest 339 ranked particles were close in cloze probability. This ranking scheme left a final 340 set of 40 plausible items fulfilling the criteria of the experiment. Cloze 341 probability and entropy statistics are summarised in Table 1. 342

To create the violation conditions, two German native speakers selected 343 
Table 1. Cloze probability and entropy summary statistics for Experiment 1.

\begin{tabular}{|c|c|c|c|}
\hline Condition & $\begin{array}{l}\text { Cloze probability } \\
\text { target particle } \\
\text { Mean }[95 \% \mathrm{CrI}]\end{array}$ & $\begin{array}{c}\text { Difference between } \\
\text { 1st- and 2nd-best completion } \\
\text { Mean }[95 \% \mathrm{CrI}]\end{array}$ & $\begin{array}{c}\text { Entropy } \\
\text { target particle } \\
\text { Mean }[95 \% \mathrm{CrI}]\end{array}$ \\
\hline 1-pa & $0.90[0.74,1.00]$ & $0.73[0.64,0.85]$ & $0.32[0.31,0.33]$ \\
\hline $2+$ particle & $0.54[0.53,0.56]$ & $0.29[0.16,0.39]$ & $0.81[0.80,0.81]$ \\
\hline
\end{tabular}

1st- and 2nd-best completions refer to the highest and second-highest cloze particles at the target site.

particles that were not integrateable into the sentence context, including illicit ${ }_{344}$ verb-particle combinations. No participant in the cloze test produced any of $\quad 345$ these illicit particles. The same particle was used in both violation sentences 346 within each item. The 40 critical sentences used in the analysis were split into $\quad 347$ four lists in a Latin square design, such that each participant only saw one of 348 the four conditions for each critical item and thus a total of 10 items per $\quad 349$ condition. Critical sentences were pseudo-randomly interspersed with 98 filler 350 sentences. Sixty-two filler sentences contained plausible sentences in a variety of 351 lengths and tenses, some of which contained non-separated particle verbs (e.g. $\quad 352$ hochgefahren, switched on). The remaining 36 fillers were particle verb 353 sentences from the cloze test that did not fulfill the 1 - vs. $2+$ particle criteria. In 354 total, each participant saw a total of 138 sentences. The ratio of plausible to 355 implausible sentences was approximately 3:1. The order of presentation of 356 sentences within each list was pseudo-randomised via the presentation software. 357 Each sentence was followed by a yes/no question appeared that probed different 358 regions of the sentence; for example, the question for example (2) above was 359 "Verlief der Vortrag ungestört?" did the lecture proceed uninterrupted?. 360

\section{$\begin{array}{ll}\text { Procedure } & 361\end{array}$}

Participants sat in a shielded EEG cabin approximately $60 \mathrm{~cm}$ from a $56 \mathrm{~cm} \quad 362$ presentation screen. The experimental paradigm was built and presented using 363 
Open Sesame [59]. Each experimental session began with an instruction screen 364 advising participants that they would read sentences presented word-by-word $\quad 365$ and that after each sentence, they would answer a question using a video game 366 controller. Participants were instructed to answer as quickly and accurately as 367 possible. Each experimental session began with four practice trials. 368

Each trial in the experiment began with a $500 \mathrm{~ms}$ fixation cross in the centre 369 of the screen followed by a blank screen jittered with a mean of $1000 \mathrm{~ms}$ and 370 standard deviation $250 \mathrm{~ms}$. Each sentence was presented word-by-word for a 371 duration of $190 \mathrm{~ms}$ per word plus $20 \mathrm{~ms}$ for each letter. The target word was $\quad 372$ always presented for $700 \mathrm{~ms}$ regardless of length. The inter-word interval was 373 $300 \mathrm{~ms}$. A comprehension question appeared after each sentence and was $\quad 374$ answered via the video game controller, which triggered the next trial. Breaks 375 were offered after every 30 sentences. The testing session including EEG setup 376 lasted approximately two hours. 377

\section{EEG recording and preprocessing 378}

The EEG recordings were made in the Department of Linguistics at the 379 University of Potsdam, Germany, in a purpose-built, electro-magnetically 380 shielded EEG cabin using a 32-lead system and electrodes arranged on the head 381 based on the international 10-20 system [60]. Electrode impedances were kept 382 below $10 k \Omega$ throughout the experiment. EEG was recorded at a sampling rate 383 of $512 \mathrm{~Hz}$ and online filtered with a low-pass filter of $138 \mathrm{~Hz}$ and using the left $\quad 384$ mastoid as a reference. 385

Raw EEG recordings were downsampled offline in BrainVision Analyzer 2, 386 Version 2.1.2, to $500 \mathrm{~Hz}$ for ease of processing and interpretation. Zero phase $\quad 387$ shift IIR Butterworth filters were applied with a low-pass cut-off at $0.01 \mathrm{~Hz} \quad 388$ (order of 2, time constant of 15.92) and a high-pass cutoff at $30 \mathrm{~Hz}$ (order of 2, $\quad 389$ no time constant). A notch filter was applied at $50 \mathrm{~Hz}$. The full recording was 390 then segmented into epochs from sentence onset to question onset. Ocular 391 
correction using restricted Infomax was applied to the sentence epochs using $\quad 392$ automatic independent component analysis (ICA) with a meaned slope 393 algorithm. The reference electrodes were two electrodes placed at the left outer 394 canthus and above the left eye to record horizontal and vertical eye movements, 395 respectively. The bound number of blinks was 60 with a convergence bound of 396 $10^{-7}$. The number of ICA steps was 512. Components were found using sum of 397 squared correlations with the horizontal and vertical ocular electrodes $61-63] . \quad 398$ The total value to delete was $30 \%$. 399

The EEG was then re-referenced to the average of the two mastoids. The ${ }_{400}$ corrected segments were further segmented into $1200 \mathrm{~ms}$ epochs representing a $\quad 401$ period of $200 \mathrm{~ms}$ before the onset of the target word (the particle), and $1000 \mathrm{~ms} \quad 402$ after onset. EEG segments with muscle artefact or irreparable eye-blink or $\quad 403$ movement artefact were automatically marked for $200 \mathrm{~ms}$ before and after each $\quad 404$ respective artefact, defined as exceeding: $\quad 405$

1. a maximum voltage step of more than $50 \mu V \quad 406$

2. a maximum absolute difference of $200 \mu V$ in a $100 \mathrm{~ms}$ interval 407

3. a minimum amplitude of $-100 \mu V \quad 408$

4. a maximum amplitude of $100 \mu V \quad 409$

5. a minimum low activity of $1 \mu V$ in a $100 \mathrm{~ms}$ interval. 410

Marked segments were then visually inspected and discarded if they indicated 411 muscle artefact or a technical issue. This resulted in the exclusion of $6.20 \%$ of $\quad{ }^{412}$ the 1000 target trials used in the statistical analysis. A further $0.50 \%$ of trials $\quad{ }^{413}$ were excluded due to question response times over 10 seconds (indicating a $\quad{ }_{414}^{414}$ technical problem) and $0.30 \%$ were not recorded due to experimenter error. The ${ }_{415}$ data were then exported and baseline-corrected in R using the package eeguana ${ }_{416}$ \begin{tabular}{lll}
\hline 64$].$ & 417
\end{tabular} 
A linear mixed effects model with full variance-covariance matrices to account $\quad{ }_{419}$ for the individual variability of subjects and items was fit to data from the $\quad{ }_{420}$ violation conditions (b) and (d) using the brms package for $\mathrm{R}$ [65]. The ${ }_{421}$ dependent variable for the N400 was mean amplitude across the electrodes $\mathrm{Cz}, \quad{ }_{422}$ $\mathrm{Pz}, \mathrm{CP} 1$, and CP2 in the 250-500 ms time window. The dependent variable for ${ }_{423}$ the PNP was mean amplitude across the electrodes Fz, FC1, and FC2 in the $\quad{ }_{424}$ 600-900 ms window. For Experiment 1, these regions were chosen post hoc after ${ }_{425}$ we deviated from the pre-registered single-electrode analysis (see S1 Appendix 426 and S2 Appendix. The regions were generally consistent with the distribution ${ }_{427}$ of the N400 and PNP observed in the predictive processing literature [32, 33, 66, 428 although some studies observe a more anterior PNP [33,36]. Since they were ${ }_{429}$ chosen post hoc, the regions of interest in Experiment 1 were used as $\quad 430$ pre-registered regions in Experiment $2 . \quad 431$

The predictor 'number of plausible particles' was effect contrast coded to $\quad{ }_{432}$ reflect our expectation for larger ERPs at 1-particle violations: 0.5 (1-particle), 433 $-0.5(2+$ particle $)$. The decision to use a categorical predictor rather than raw $\quad{ }_{434}$ entropy was made because the precise functional relationship between entropy $\quad 435$ and ERP amplitude was not known, and we felt unable to safely assume it was ${ }_{436}$ $\begin{array}{ll}\text { linear. } & 437\end{array}$

To constrain the model to plausible values and avoid overfitting, we placed $\quad 438$ regularising priors of $N(0,1)$ on each parameter 67,70 . This prior with a 439 normal distribution centered on zero indicates to the model that, for example, $\quad{ }_{440}$ the effect of the number of plausible particles will most likely be small or zero, ${ }_{441}$ with either a positive or negative sign, and has a $95 \%$ probability of lying $\quad 442$ between -2 and $2 \mu V$. 443

To quantify how much evidence we had for any N400 and PNP effect, we 444 conducted Bayes factor analyses comparing models with the predictor number of $\quad{ }_{445}$ 
particles (M1) versus a reduced model without this predictor $(\mathrm{M} 0)$, i.e. $B F_{10}$. A ${ }_{446}$ Bayes factor of approximately 1 would indicate no evidence in favour of either $\quad{ }_{447}$ model. As the Bayes factor increases over 3, evidence strengthens in favour of ${ }_{448}$ M1 71,72. As the Bayes factor decreases under 0.3, evidence strengthens in ${ }_{449}$ favour of M0. Since the Bayes factor is sensitive to the choice of prior [73, 74], 450 we conducted a sensitivity analysis by computing Bayes factors for a range of $\quad{ }_{451}$ priors to determine how these affected our conclusions 75,76 . We used a range ${ }_{452}$ of priors that assumed a priori effect sizes ranging from small, between -0.2 and $\quad 453$ $0.2 \mu \mathrm{V}$, to large, between -10 and $10 \mu \mathrm{V}$. 454

To confirm that the violation particles elicited an N400 and PNP relative to 455 their plausible counterparts in line with previous studies [77, we fit the above 456 models to all conditions (a-d) and added the predictor 'plausibility'. Plausibility ${ }_{457}$ was effect contrast coded to reflect our expectation for larger ERPs in the $\quad{ }_{458}$ implausible conditions: 0.5 (implausible), -0.5 (plausible) and a Bayes factor was ${ }_{459}$ computed at a prior of $N(0,1)$. Since the plausible particles differed in cloze $\quad 460$ probability and identity from each other and from the violation particles, we $\quad 461$ used this analysis as a sanity check only and did not conduct any further $\quad 462$ $\begin{array}{ll}\text { analyses. } & 463\end{array}$

$\begin{array}{ll}\text { Results } & 464\end{array}$

Mean amplitude relative to the onset of the target particle is plotted in Figure 1. 465 In Figure 1A-B, the 250-500 ms window of the ERP shows a strong negative $\quad 466$ deflection over the posterior scalp for the implausible conditions relative to the $\quad 467$ plausible conditions, consistent with an N400. Implausible particles also elicited ${ }_{468}$ a large positive deflection in the $600+\mathrm{ms}$ time window relative to plausible $\quad{ }_{469}$ particles over the posterior scalp, consistent with a P600. Figure 1C-D shows a 470 positive deflection in the post-N400 period of the ERP (i.e. after $500 \mathrm{~ms}$ ) in $\quad{ }_{471}$ fronto-central electrodes consistent with a PNP in the 1-particle condition $\quad 472$ relative to the $2+$ particle condition and relative to the plausible conditions. $\quad 473$ 
At implausible particles, the N400 was more negative in the 1-particle condition $\quad 475$ than in the $2+$ particle condition and the mean of the posterior was $-0.34 \mu \mathrm{V} \quad 476$ with a $95 \%$ credible interval $(\mathrm{CrI})$ of $[-1.10,0.39] \mu V$ (Figure $2 \mathrm{~A})$. The Bayes $\quad 477$ factor at a prior of $N(0,1)$ favoured neither the null or alternative hypotheses $\quad 478$ about an entropy-related difference in the N400, $B F_{10}=0.57$. The sensitivity $\quad{ }_{479}$ analysis suggested there was moderate evidence against a priori hypothesised $\quad 480$ large effect sizes, but was inconclusive about small effect sizes (Figure 2B).There ${ }^{481}$ was strong evidence that the N400 was more negative for implausible than $\quad{ }_{482}$ plausible particles, $\hat{\beta}=-1.09 \mu V,[-1.69,-0.48], B F_{10}=465$.

\section{Analysis of the anterior post-N400 positivity (PNP) 484}

At implausible particles, the posterior mean indicated more positive amplitude $\quad 485$ in the 1 - vs. the $2+$ particle condition, $\hat{\beta}=0.74 \mu \mathrm{V}, 95 \% \operatorname{CrI}[-0.25,1.77] \mu \mathrm{V} \quad{ }_{486}$ (Figure $2 \mathrm{C}$ ). However, the Bayes factor at a prior of $N(0,1)$ favoured neither the ${ }_{487}$ null or alternative hypotheses about an entropy-related effect on the PNP, $\quad 488$ $B F_{10}=1.50$. The sensitivity analysis suggested effect sizes of approximately $\quad{ }_{489}$ $1 \mu V$ were more plausible than smaller or larger effects, although the Bayes $\quad 490$ factors even at $1 \mu V$ were inconclusive (Figure 2D). The PNP was more positive 491 for implausible than plausible particles, although the Bayes factor suggested only ${ }^{492}$ anecdotal evidence for this conclusion, $\hat{\beta}=0.49 \mu V,[-0.29,1.24], B F_{10}=2.69 . \quad{ }^{493}$

\section{Interim discussion}

We compared sentence pairs where either a single verb particle or a small set of 495 verb particles were plausible continuations given the context. We hypothesised 496 that lower entropy would encourage commitment to a lexical prediction in the $\quad{ }_{497}$ 1-particle condition, and that violating this prediction would be more costly $\quad{ }_{498}$ relative to the $2+$ particle condition where higher uncertainty discouraged $\quad 499$ 
A
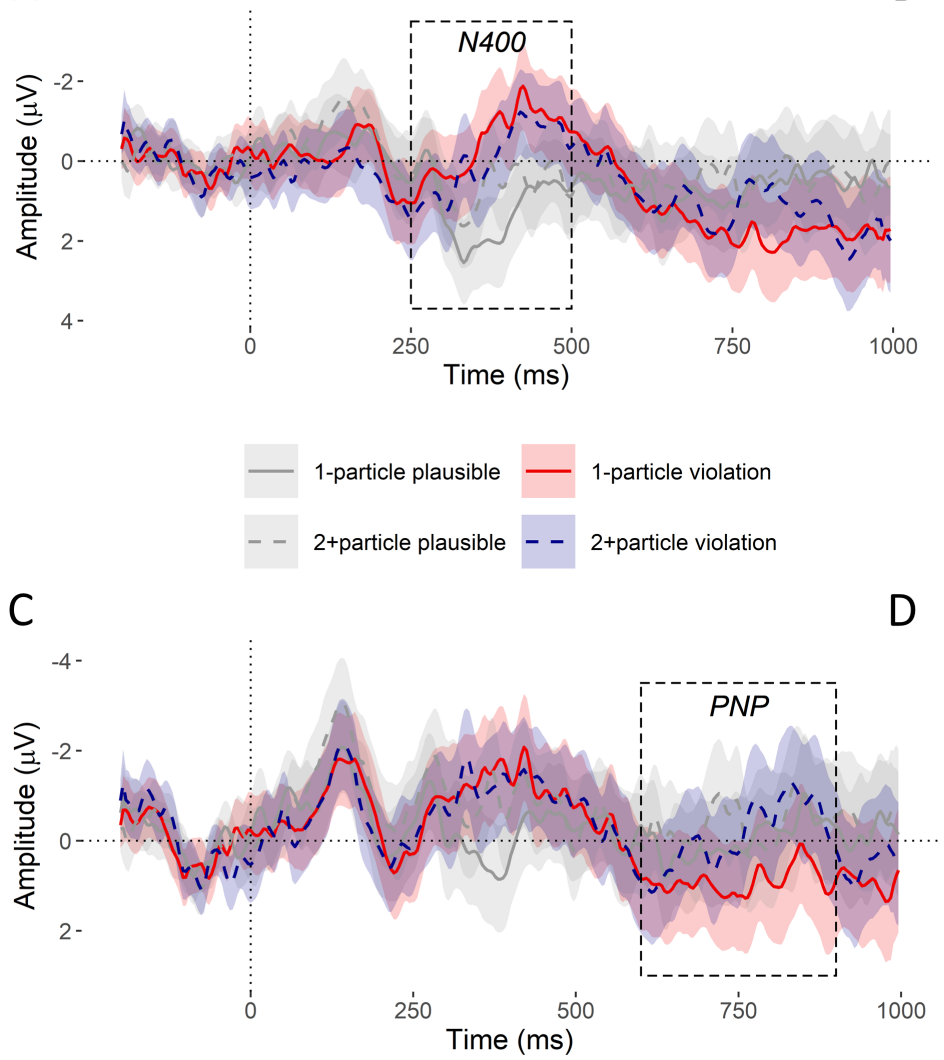

B
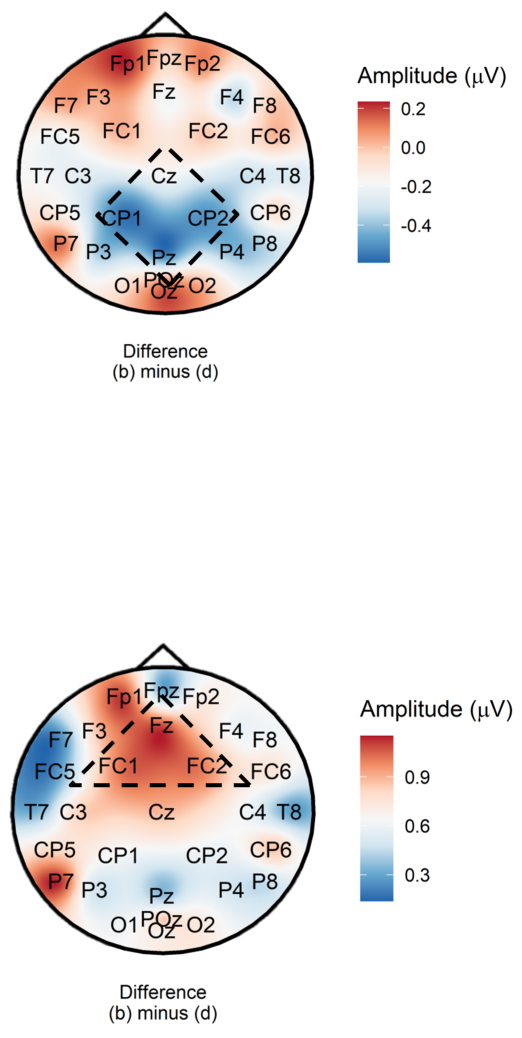

Fig 1. Experiment 1: ERP results. A. Average amplitude following stimulus onset shows an N400 in the posterior region of interest $(\mathrm{Cz}, \mathrm{CP} 1, \mathrm{CP} 2$, $\mathrm{Pz}$ ) for violations of both the 1-particle (red) and 2+particle (blue) conditions relative to the plausible (grey) conditions. Ribbons show $95 \%$ confidence intervals. These intervals were calculated by fitting a linear model of the form amplitude $\sim$ condition with by-subject and by-item intercept adjustments at each timestep of the ERP recording and extracting the standard error of the relevant conditions. The analysed N400 time window is overlaid. B. A voltage subtraction map of the N400 time window shows a small difference in amplitude between the 1-particle (a) and 2+particle (b) conditions in the region of interest (dashed square). C. A PNP for the 1-particle condition is seen in the anterior region of interest (Fz, FC1, FC2) 600-900 ms window after stimulus onset, but not in the $2+$ particle or plausible conditions. Ribbons show $95 \%$ confidence intervals. D. A voltage subtraction map of the PNP time window suggests amplitudes in the 1-particle condition were more positive in the mid-frontal region (dashed triangle) than in the $2+$ particle condition.

prediction. We predicted that this cost would be reflected in a larger N400 and 500 PNP. Contrary to this prediction, evidence about the effect of entropy on both 501 the N400 and PNP was inconclusive. The comparison of plausible versus 
A

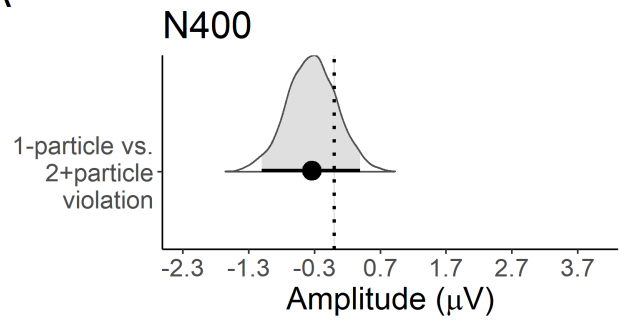

C

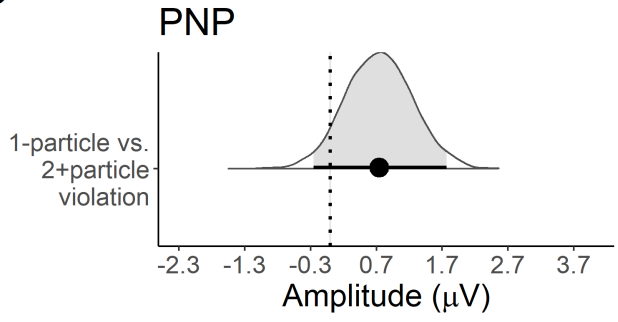

B
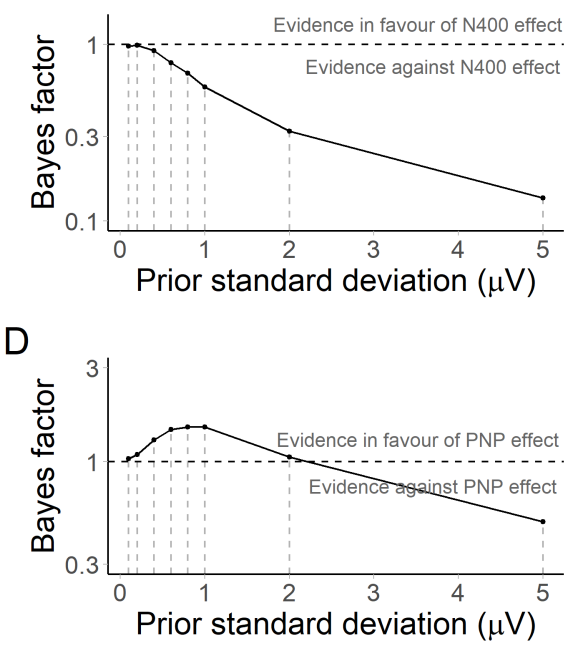

Fig 2. Experiment 1: Posterior estimates and Bayes factor analysis for the planned and exploratory comparisons. A. The posterior distribution represents the estimated change in N400 amplitude associated with violations in the 1 -particle condition relative to the $2+$ particle condition. The point and errorbar reflect the posterior mean and $95 \%$ credible interval. B. Bayes factors computed under a range of priors comparing models with and without a predictor for the 1- vs. 2+particle condition manipulation. A Bayes factor of 1 indicates no evidence in favour of either model. Bayes factors below 1 indicate evidence against a difference in N400 amplitude associated with the manipulation. C. Posterior distribution of the difference in PNP amplitude between the violation conditions. D. Bayes factors under a range of priors for the PNP effect.

implausible particles elicited a difference in both component in line with the $\quad 503$ typical plausibility effect observed in previous research, including research on $\quad 504$ German particle verbs [77], confirming that the experiment worked in principle, 505 although evidence for the effect on the PNP was less conclusive than expected. 506

While Bayes factor evidence for the effect of entropy on the N400 was 507 inconclusive, the magnitude of the effect was small, which could be considered $\quad 508$ consistent with the null hypothesis of lexical access and probabilistic update $\quad 509$ accounts $42,43,47]$. Under these accounts, the N400 at zero-probability words 510 should not be affected by uncertainty caused by multiple activated words. One ${ }_{511}$ caveat to this conclusion is that, if there were truly no N400 effect, one could $\quad 512$ have expected stronger support for the null hypothesis from the Bayes factor $\quad 513$ 
analysis, especially since the cloze probability manipulation was able to yield $\quad 514$ strong evidential support. One reason that the Bayes factor may not have been 515 able to distinguish between the null and alternative hypotheses is that the study 516 was not sufficiently powered to detect or rule out an effect of entropy. While the 517 study had a sample size of 50-larger than many ERP studies in the 518 literature - a small number of critical trials (10 per participant) likely led to a $\quad{ }_{519}$ large amount of noise in the averaged ERP data. $\quad 520$

Bayes factor evidence for the PNP was also inconclusive, but the effect size $\quad{ }_{521}$ was larger and consistent in sign with previous findings on the effect of 522 constraint (larger PNP for violations in high-constraint settings) [32, 33, 36. 523 This may suggest that lower entropy led to a prediction in the 1-particle $\quad 524$ condition, eliciting a higher suppression cost once this prediction was violated. $\quad 525$ Higher entropy in the $2+$ particle condition may have discouraged predictions, $\quad{ }_{526}$ avoiding this cost. However, again, one could have expected stronger evidence $\quad 527$ from the Bayes factor. The findings would therefore also be consistent with a $\quad{ }_{528}$ null effect in line with current PNP accounts suggesting that the component $\quad 529$ should not be sensitive to entropy if the input is implausible 33, 57. 530

One possible explanation for the weak Bayes factors is that variability was $\quad{ }_{531}$ introduced into the data by individual differences in dealing with the 532 implausible verb particles. Particles are more syntactically and semantically $\quad 533$ ambiguous than the implausible nouns used in previous PNP research and $\quad 534$ participants may have tried to reanalyse them in different ways, while others $\quad 535$ made no attempts at revision. For example, an implausible verb particle such as 536 make [a story] *on could potentially be revised as a preposition make [a story ${ }_{537}$ [on the war in Iraq]]... The fact that a large posterior P600 was also elicited by ${ }_{538}$ the violations may indicate that such a syntactic reanalysis was attempted. $\quad 539$

However, any conclusions from Experiment 1 are limited by a small number $\quad 540$ of critical trials, even though the sample size was relatively large for an ERP $\quad{ }_{541}$ study. To test whether a larger study would provide more conclusive evidence $\quad 542$ 
for the PNP effect and against the N400 effect, we used the results of 543

Experiment 1 to pre-register a second experiment with double the number of $\quad 544$ participants and more than double the number of experimental sentences. $\quad 545$

\section{Experiment 2}

Experiment 2 sought to replicate the findings of Experiment 1 in a larger $\quad 547$ sample, using a larger number of experimental items. Since Experiment 1 already confirmed the presence of the expected ERP components at plausible vs. 549 implausible particles, to further increase the probability of detecting an effect, $\quad 550$ we removed the plausible conditions to create a two-condition design. This 551 meant that the implausible conditions could be split into two rather than four $\quad{ }_{552}$ lists, doubling the number of critical trials each participant would see. In line $\quad{ }_{553}$ with Experiment 1, we expected that we would not see a difference in N400 $\quad 554$ amplitude between 1-particle and 2+particle violations, but that the PNP might 555 be larger in the 1-particle than the 2+particle condition. Experiment 2 was $\quad 556$ pre-registered at https://osf.io/y6k2d; see also S1 Appendix. 557

Methods

$\begin{array}{ll}\text { Participants } & 559\end{array}$

Recruitment, inclusion, and exclusion criteria were identical to those in $\quad 560$

Experiment 1. To estimate a suitable sample size for Experiment 2, we 561 conducted a power analysis using the data from Experiment 1. Although $\quad 562$ Bayesian analysis was used for the main analysis, we conducted a frequentist $\quad 563$ power analysis as it was computationally lighter and serves as a quick ballpark 564 estimate of the sample size needed for obtaining high-precision estimates $[78$. 565 The power analysis suggested that even with 300 participants we would only $\quad 566$ achieve around $70 \%$ power. Due to resource constraints, it was only feasible to $\quad 567$ collect data from around 100 participants. We therefore set a goal of $100 \quad 568$ 
participants as the maximum feasible sample size and redid the power analysis, 569 which estimated around $50 \%$ power at this sample size. While likely to be $\quad 570$ underpowered, our goal with Experiment 2 was to provide higher-precision $\quad 571$ estimates of the effects observed in Experiment 1 using an improved design, and 572 to see to what extent doubling the size of the experiment would improve our $\quad 573$ ability to quantify evidence for our hypotheses. This information is useful to 574 future evidence synthesis and in determining how one might approach future $\quad 575$ experiments. In total, 115 participants were recruited, 4 of whom were excluded 576 as they did not meet the inclusion criteria. A further 11 were excluded due to $\quad 577$ technical problems with the EEG recording. This left a total of 100 participants 578 (24 male), with a mean age of 24 years (range $=18$ to 35 years, $\mathrm{SD}=4$ years). $\quad{ }^{579}$

\section{Materials}

Having established in Experiment 1 that the implausible particles did elicit the ${ }_{581}$ target ERP components relative to their plausible counterparts, for 582 Experiment 2 we increased power by having participants see one of the two 583 violation conditions from each item rather than one of the four total conditions. 584 The plausible conditions were replaced by a length-matched sentence for each $\quad 585$ item that contained a separated verb-particle dependency with a plausible $\quad 586$ particle. This filler served to maintain the ratio of plausible to implausible $\quad 587$ particle verb sentences, and to serve as a sanity check. These fillers were not $\quad 588$ analysed as they were otherwise unmatched with the implausible sentences. $\quad 589$ Each participant thus saw either condition (b) or (d), and the length-matched, 590 plausible filler from each item. 591

The sentences from Experiment 1 were re-used, plus 11 new sentences 592 constructed using the same procedure as for Experiment 1. The new sentences 593 were selected from a pool of 20 cloze-tested with 30 native German speakers $\quad 594$ (mean age 24 years, SD 5 years, range 18-38 years). To create an even number of 595 sentences, one of the new sentences replaced the lowest-ranked of the 40 items $\quad 596$ 
from the original cloze test. This gave a total of 50 critical items, making a total ${ }_{597}$ of 50 items (25 per condition) analysed in Experiment 2. Cloze probabilities and 598 entropy are summarised in Table 2.

Table 2. Cloze probability summary statistics for Experiment 2.

\begin{tabular}{|c|c|c|c|}
\hline Condition & $\begin{array}{l}\text { Cloze probability } \\
\text { target particle } \\
\text { Mean }[95 \% \mathrm{CrI}]\end{array}$ & $\begin{array}{c}\text { Difference between } \\
\text { 1st- and 2nd-best completion } \\
\text { Mean }[95 \% \mathrm{CrI}]\end{array}$ & $\begin{array}{c}\text { Entropy } \\
\text { target particle } \\
\text { Mean }[95 \% \mathrm{CrI}]\end{array}$ \\
\hline 1-particle & $0.89[0.73,1.00]$ & $0.73[0.64,0.84]$ & $0.31[0.30,0.32]$ \\
\hline $2+$ particle & $0.53[0.52,0.55]$ & $0.28[0.15,0.38]$ & $0.81[0.80,0.81]$ \\
\hline
\end{tabular}

1st- and 2nd-best completions refer to the highest and second-highest cloze particles at the target site.

In addition to the 50 target items and 50 matched fillers presented to 600 participants, 108 general filler sentences were randomly interspersed. These were 601 62 of the fillers from the Experiment 1 that did not contain separated particle $\quad 602$ verbs, plus 46 new fillers, also not containing separated particle verbs. Overall, 603 each participant saw a total of 208 sentences during the testing session. The ${ }_{604}$ ratio of plausible to implausible sentences was 4:1. Participants again answered 605 a yes/no question after each sentence. 606

$\begin{array}{ll}\text { Procedure, EEG recording, preprocessing } & 607\end{array}$

The data collection procedure and preprocessing were identical to that of $\quad 608$

Experiment 1. EEG data cleaning resulted in the exclusion of $12.26 \%$ of the $\quad{ }^{609}$ 5000 target trials due to artefact. A further $0.60 \%$ were excluded due to $\quad 610$ question response times over 10 seconds and $0.32 \%$ were not recorded due to $\quad{ }_{611}$

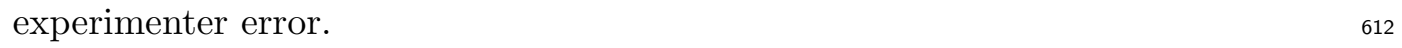

$\begin{array}{ll}\text { Analysis } & 613\end{array}$

The analysis comparing the two violation conditions for Experiment 2 was $\quad 614$ identical to that of Experiment 1. The plausible filler was not analysed as there ${ }_{615}$ were too many differences between these fillers and the critical items. 616 


\section{Results}

Figure3A-B show that the 250-500 ms window of the ERP showed a strong $\quad 618$ negative deflection in the implausible conditions relative to the plausible filler, $\quad{ }_{619}$ consistent with an N400. A posterior P600 was elicited by implausible particles 620 in the $600+\mathrm{ms}$ time window. Figure 3C-D also shows a positive deflection in ${ }_{621}$ the post-N400 period of the ERP (i.e. after $500 \mathrm{~ms}$ ) in fronto-central electrodes ${ }_{622}$ consistent with a PNP, however this time in both the 1- and 2+particle 623 conditions relative to the plausible filler, and most positive in the $2+$ particle $\quad{ }_{624}$ condition.

\section{Analysis of the N400}

As for Experiment 1, there was a small between-condition difference in N400 $\quad{ }_{627}$ amplitude at implausible particles, $\hat{\beta}=-0.22 \mu V$, 95\% CrI [-0.66, 0.22] Figure $\quad{ }^{628}$ 4 A,but the Bayes factor at a prior of $N(0,1)$ favoured the null hypothesis that ${ }_{629}$ there was no effect of entropy on the N400, $B F_{10}=0.35$. The sensitivity 630 analysis indicated more conclusive evidence against a priori hypothesised large $\quad 631$ effect sizes than in Experiment 1, but was still inconclusive about small effect $\quad{ }_{632}$ sizes despite the increased sample size Figure 4B 633

\section{Analysis of the anterior post-N400 positivity (PNP) 634}

The PNP effect at implausible particles appeared to have reversed relative to $\quad{ }_{635}$ Experiment 1, with a more positive waveform in the $2+$ than the 1-particle $\quad{ }_{636}$ condition. The posterior mean indicated a small difference in amplitude between $\quad 637$ the violation conditions, $\hat{\beta}=-0.40 \mu V, 95 \% \mathrm{CrI}[-0.89,0.12]$ (Figure 4C). $\quad{ }_{638}$ However, the Bayes factor at a prior of $N(0,1)$ favoured neither the null or $\quad 639$ alternative hypotheses about an effect of entropy on the PNP, $B F_{10}=0.84$. The ${ }_{640}$ sensitivity analysis this time indicated evidence against a priori hypothesised $\quad{ }_{641}$ large effect sizes, but was even more inconclusive about smaller effect sizes than $\quad 642$ 
A

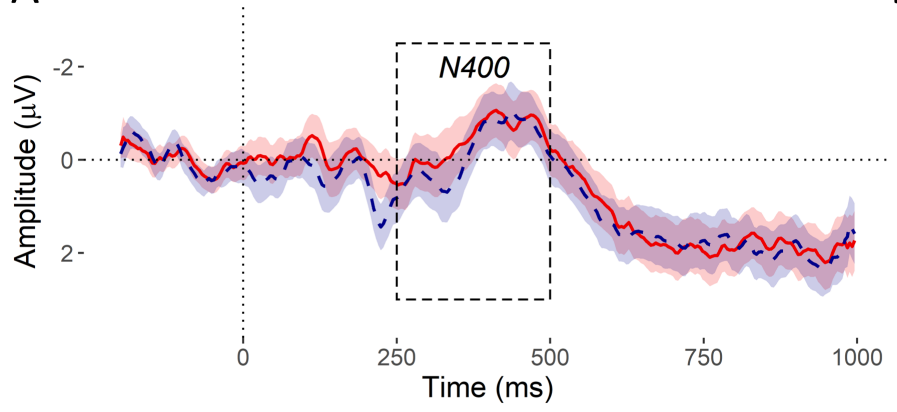

1-particle violation - - $2+$ particle violation
B

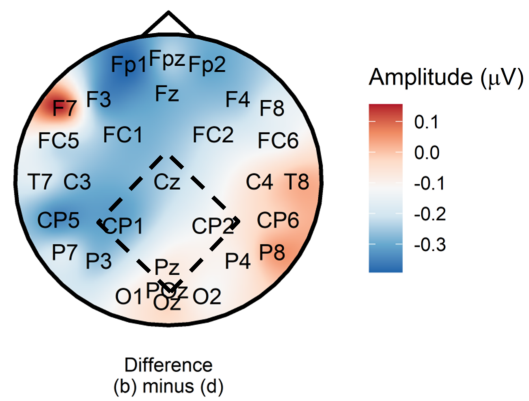

D

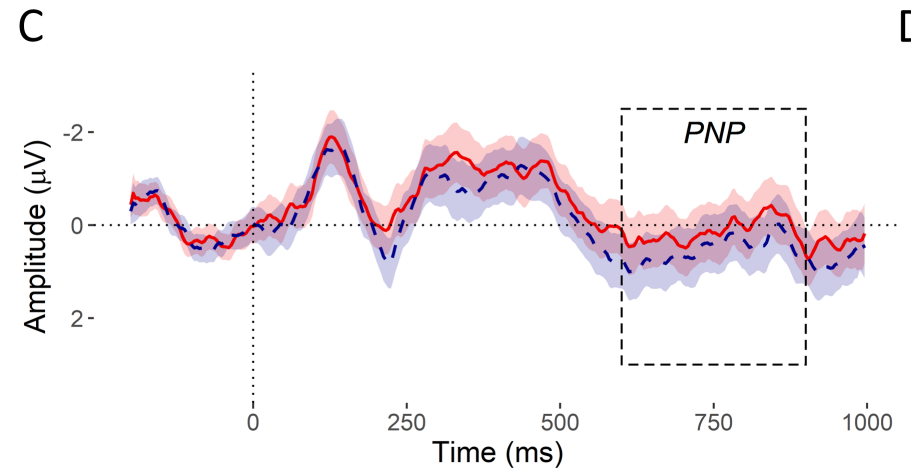

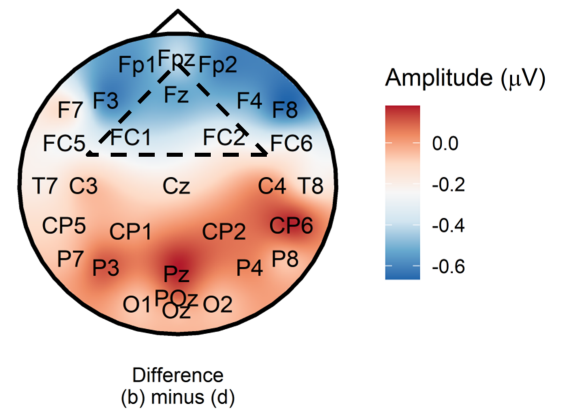

Fig 3. Experiment 2: ERP results. A. ERP waveforms showing an N400 in 1-particle (red) and 2+particle (blue) in the posterior region of interest $(\mathrm{Cz}$, CP1, CP2, Pz). Ribbons show 95\% confidence intervals calculated as for Figure 1. The pre-registered time window of the N400 is overlaid. B. The voltage subtraction plot of the difference in amplitude between 1- and +particle violations in the N400 time window indicates very little difference in amplitude between the two conditions in the posterior region of interest (dashed square). C. ERP waveforms in anterior (Fz, FC1, FC2) region suggest that the most positive waveform in the pre-registered PNP time window (overlaid) was for $2+$ particle violations. Ribbons show $95 \%$ confidence intervals. D. The voltage subtraction plot of the difference in amplitude between 1- and 2+particle violations in the PNP time window suggests amplitude was more positive for $2+$ particle violations in the anterior region of interest (dashed triangle).

for Experiment 1 (Figure 4D).

In summary, despite the relatively large amount of data and a clean manipulation of entropy, no conclusive effect on the N400 or PNP was detected. ${ }^{645}$ Visual inspection of the ERPs did not point to any other effects of the $\quad 646$ manipulation. 
A

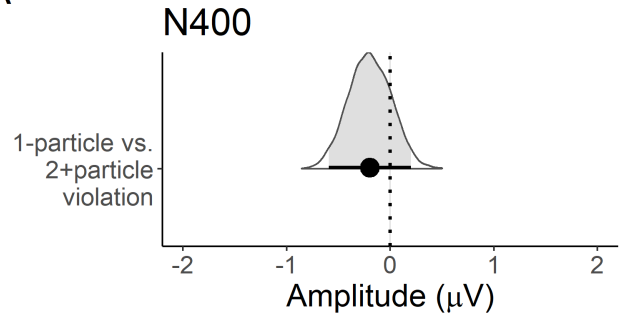

C

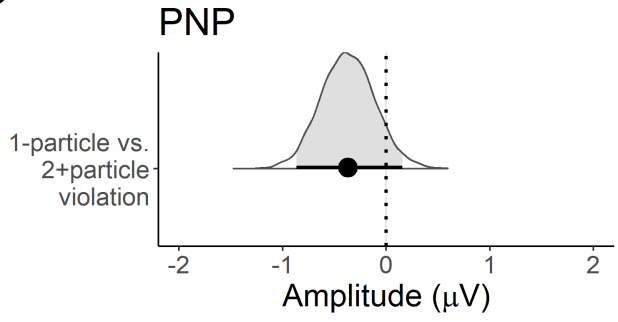

B
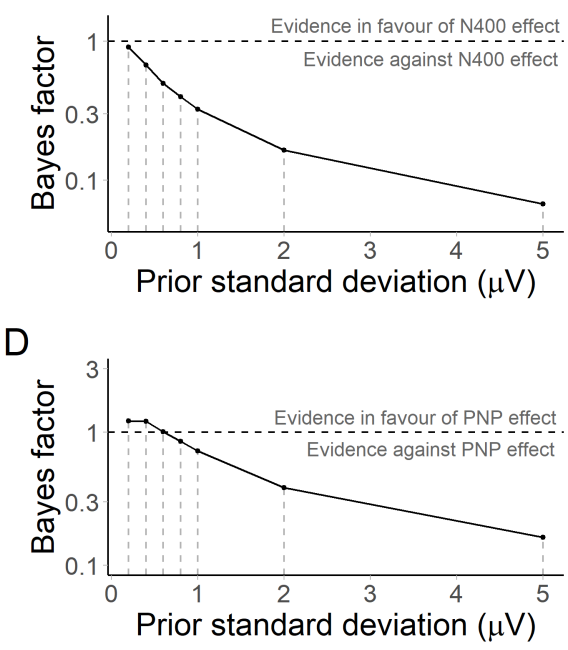

Fig 4. Experiment 2: Posterior estimates and Bayes factor analysis for the planned comparisons. A. Posterior distribution of the estimated difference in N400 amplitude associated with 1-particle condition vs. 2+particle violations. The mean and $95 \%$ credible interval are overlaid. B. Bayes factors computed under a range of priors comparing models with and without a predictor for the 1- vs. 2+particle condition manipulation. A Bayes factor of 1 indicates no evidence in favour of either model. Bayes factors below 1 indicate evidence against a difference in N400 amplitude associated with condition. C. Posterior distribution of the difference in PNP amplitude between the violation conditions. D. Bayes factors under a range of priors for the PNP effect.

\section{Discussion}

Experiment 2 replicated the N400 findings observed in Experiment 1, with both ${ }_{649}$ violation particles eliciting an N400 of a similar amplitude regardless of whether 650 one or more than one particle had been plausible in the context. Interestingly, ${ }_{651}$ the Bayes factor analysis did not become more conclusive with the increased $\quad 652$ sample size and number of trials, although the data were sufficient to rule out $\quad 653$ large effect sizes. The increased sample size and number of experimental stimuli 654 may therefore still have been too small to yield conclusive evidence. This $\quad 655$ possibility is discussed further in the General discussion, as well as the relevance ${ }_{656}$ of the findings with respect to current models of the N400. 657

With respect to the PNP, the source of the reversal in effect direction relative 658 
to Experiment 1 was unclear. The difference in sign could reflect oscillation $\quad 659$ around a true mean of zero with a wide standard deviation. A true mean of zero $\quad 660$ would be in line with current accounts of the PNP, which suggests that 661 implausible words such as our particles should not elicit a entropy-based $\quad 662$ difference in amplitude. However, the inconclusive Bayes factor may suggest $\quad 663$ that the data were too noisy to distinguish between the tested hypotheses at a ${ }_{664}$ priori hypothesised small effect sizes, even though sensitivity analyses indicated 665 $\begin{array}{ll}\text { evidence against large effect sizes. } & 666\end{array}$

\section{$\begin{array}{ll}\text { General discussion } & 667\end{array}$}

In two experiments, we investigated whether uncertainty, as measured by $\quad 668$ entropy, would influence readers' willingness to predict the lexical identity of an ${ }_{669}$ upcoming particle in a long-distance verb-particle dependency. Experiment $1 \quad 670$ suggested that predictions had been made when certainty was high, based on $\quad 671$ the apparent cost of suppressing the disconfirmed sentence representation $\quad 672$ indicated by a larger anterior post-N400 positivity (PNP). However, statistical 673 evidence was inconclusive and the effect was not replicated in the larger 674 Experiment 2. In Experiment 2, entropy did not appear to have an effect on $\quad 675$ processing difficulty at the violations, although statistical evidence was again $\quad 676$ inconclusive. We interpret the findings as related to how readers dealt with the 677 $\begin{array}{ll}\text { implausible verb particle. } & 678\end{array}$

\section{$\begin{array}{ll}\text { Implausible input and the N400 } & 679\end{array}$}

The lack of conclusive evidence for an entropy-based N400 difference could be $\quad{ }_{680}$ interpreted as being consistent with current accounts of the N400, which would 681 not have predicted a difference [43, 47]. This would further suggest that the ${ }_{682}$ highest-cloze-probability definition of constraint used in the studies on which $\quad 683$ these accounts are based is sufficiently similar to entropy, and that the $\quad 684$ 
distribution of co-activated words is not an important factor in explaining N400 685 amplitude; at least not at an implausible word. Indeed, these accounts of the $\quad 686$ N400 make no explicit predictions about how co-activated words should affect $\quad 687$ processing of a zero-probability word; however, one could have imagined that $\quad{ }_{688}$ lexical access [43 or probabilistic update 47] might be dampened by the $\quad 689$ presence of co-activations if these activations consume the same resources, or $\quad 690$ create a large amount of uncertainty in the system. Co-activated words have in 691 fact been observed to modulate amplitude of the N400 at plausible but 692 unexpected words $38,42,47,79$. One contribution of the current study may $\quad 693$ therefore be to confirm the implicit assumption of current N400 accounts that ${ }_{694}$ uncertainty caused by competing lexical representations does not affect N400 695 amplitude if the input is implausible. 696

One caveat to any conclusion about the N400 in the current study is the $\quad{ }_{697}$ inconclusive Bayes factor analysis at small effect sizes. If the N400 is truly not ${ }_{698}$ affected by constraint, then with sufficient data, one should have expected a $\quad{ }^{699}$ Bayes factor at least weakly in favour of the null hypothesis. While the data 700 were sufficient to find evidence against a priori hypothesised large effect sizes, $\quad 701$ the inconclusive evidence against small effect sizes, even in the larger 702 Experiment 2, raises several possibilities: first, that some property of the $\quad 703$ verb-particle violation differs to the types of violations in which the N400 is 704 usually studied and that this difference elicits a less clear-cut effect on the N400; 705 second, given a less clear-cut effect, that an even larger sample size would have 706 been needed to provide conclusive evidence against an entropy effect; or third, $\quad 707$ that implausible words really did elicit a small difference in N400 amplitude $\quad 708$ associated with entropy, but that the difference was too small to yield conclusive 709 evidence at the current sample size. We discuss the properties of the 710 verb-particle dependency next in relation to the PNP, in which the interaction 711 of plausibility and constraint (a proxy for entropy) has been directly tested in 712 $\begin{array}{ll}\text { previous research. } & 713\end{array}$ 
With respect to sample size, we again used a frequentist power analysis to $\quad 7_{14}$ gain a rough estimate of the sample size that would be needed for a 715 hypothetical, future experiment using the same experimental design and 716 assuming that the effect was the same size observed in Experiment 2 80. This 717 prospective power analysis suggested that thousands of participants would be $\quad{ }_{718}$ needed to achieve even $30 \%$ power, strongly suggesting that the above questions 719 are likely not answerable with this particular design. Compare this to the power 720 analysis conducted using the lower-precision estimates from Experiment 1, 721 which vastly overestimated that we could achieve around $50 \%$ power with just $\quad 722$ 100 participants.

Previous findings suggest that constraint - similar to entropy - does not modify 725 the PNP when the input is implausible 33,57. The lack of conclusive evidence 726 for an entropy effect at implausible particles in the current study may therefore 727 be consistent with the hypothesis that the PNP reflects only successful update 728 of a mental sentence representation triggered by plausible input [33]. However, 729 if the PNP is not elicited by implausible input, we should have expected $\quad 730$ conclusive evidence in favour of the null hypothesis, especially in the larger $\quad 731$ Experiment 2. It is therefore possible that something about the violation $\quad 732$ particle was different to the types of violations in which the plausibility effect on 733 the PNP has previously been studied. Specifically, the implausible particle $\quad 734$ violates expectation at a purely semantic level of representation, since any $\quad 735$ syntactic expectation for 'a particle' was satisfied. In contrast, previous PNP 736 studies have typically used verb-noun dependencies, where an implausible noun $\quad 737$ violates expectations at a number of levels of representation (e.g. animacy, 738 thematic role, semantic). This raises the possibility that implausibility affects 739 predictive processing cost in a graded way, with 'lighter' implausibility being $\quad 740$ $\begin{array}{ll}\text { simply less costly. } & 741\end{array}$ 
Alternatively, lighter violations may be more amenable to repair. In the 742 current study, repair may have been aided by the fact that the verb-particle $\quad 743$ construction denotes a more abstract concept than a noun, which may allow $\quad 744$ attempts to update the semantic representation. For example, ${ }^{*}$ carried with is $\quad 745$ not a licensed verb-particle combination, but one could perhaps more easily $\quad 746$ invent a situation to accommodate it than to accommodate an implausible noun. 747 Moreover, German particles are identical to prepositions and thus readers may 748 have attempted to revise the particle as a preposition, e.g. carried his lecture $\quad 749$ [with determination] on. The large P600 in both experiments may suggest that 750 such reanalysis was attempted $33,46,52,54,81,84$.

$\begin{array}{ll}\text { In further support of a role of dependency type in modulating whether } & 752\end{array}$ implausibility elicits the PNP, visual inspection of the ERPs in both experiments 753 suggested that implausible particles did elicit a more positive waveform in the 754 PNP window than plausible particles. Previous research in implausible nouns 755 suggests that this should not have been the case 33 57. However, the plausible 756 and implausible particles in both experiments were not matched for frequency or 757 lexical identity, and so this finding requires future investigation in a more $\quad 758$ controlled experiment. In summary, one possible explanation for the inconclusive 759 findings in both the PNP and N400, despite the large sample size, is that the 760 level of representation at which expectations are violated may be an important 761 factor in using ERPs to measure how entropy affects predictive processing. $\quad 762$

\section{Entropy and the P600}

While the P600 was not a focus of the current experiments and so was not $\quad 764$ analysed, its visual appearance in Figure 3 (Experiment 2) suggests that it was 765 not affected by entropy. This contrasts with previous studies of anomalous $\quad 766$ words, where violations of strong constraint elicited larger P600s than violations 767 of weak constraint [33]; for constraint effects at unexpected but still plausible 768 words, see 85, 86]. Kuperberg and colleagues propose that stronger constraint 769 
makes the discrepancy between expected and received input larger, leading to a 770 larger P600. This could suggest that the meaning discrepancy in the current 771

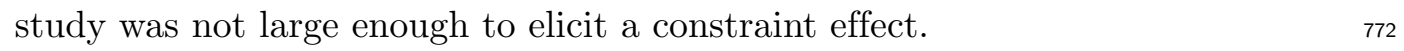

However, there is another interesting possibility: that the violation occurred 773 at different levels of representation between studies. Kuperberg and colleagues 774 violated the animacy and thematic role constraints of expected nouns. In 775 contrast, our particles violated only semantic constraints: there are no thematic 776 roles or animacy features associated with particles, and although they could be 777 reanalysed as prepositions, the morphosyntactic form of either a particle or $\quad 778$ preposition was not violated. Our P600 therefore contrasts with accounts of the 779 P600 as a discrepancy between lexical expectations and expected morphologic $\quad 780$ form 81, 85, 87, as well as with accounts associating it with syntactic 781 processing 51, 54, 88. It is perhaps more consistent with accounts of the P600 782 as reflecting syntactic reprocessing attempts [89], general error detection and 783 reanalysis process [83, 90], or integration processes [52,55, 56]. We leave to 784 future research the question of whether the difference between our and 785 Kuperberg and colleagues' violations suggest that violating different levels of $\quad 786$ $\begin{array}{ll}\text { representation affect the P600 differently. } & 787\end{array}$

\section{Alternative accounts of ERP findings at the particle 788}

Based on previous findings, we have attributed our inconclusive results to the 789 difficulty of interpreting ERPs elicited by implausible words rather than to 790 evidence that predictions were not made based on previous findings in particle 791 verbs and similar constructions $33,5,91$. However, a non-prediction account is 792 also compatible with the current findings. An integration account would propose 793 that the lexical identity of the particles was not preactivated, but rather that 794 processing was initiated once the particle was encountered in the input 92 94. 795 Being equally unintegrateable into the sentence, an equal amount of processing 796 difficulty would then be reflected in the amplitude of the ERP components. An 797 
integration account could be contradicted by examining ERP differences earlier 798 in the sentence; for example, by examining whether working memory load $\quad 799$ differed in line with whether a prediction had been made or not, as indexed by 800 the left anterior negativity (LAN) or sustained anterior negativity (SAN). 801 However, it is unclear whether the LAN is sensitive to working memory load 802 associated with lexical ambiguity as it is more often studied in relation to 803 syntactic structure building [12 14], although lexical ambiguity was found not 804 to play a role in one study 91. An investigation of these components is beyond 805 the scope of the current article, but would be worthy of future examination to 806 further disentangle prediction from non-prediction accounts. 807

A second alternative account of our findings is that of Piai et al. (2013), who 808 concluded that the lexical representations of verbs that take particles - rather $\quad{ }_{809}$ than of the particles - are maintained in working memory to facilitate their $\quad 810$ retrieval once the particle is encountered. Since the verbs should have been $\quad{ }_{811}$ equally retrievable, this would explain why no differences in ERP amplitude $\quad 812$ were observed at the particle site. Our findings would also support the idea that 813 a syntactic placeholder for a particle was posited, in the same way that gaps are 814 posited in filler-gap dependencies [12 14], but that the lexical features of the $\quad 815$ required particle were not activated until the particle was encountered. As 816 mentioned, there is some evidence from reading time studies to suggest that $\quad 817$ entropy may influence the preactivation of long-distance lexical information $\quad{ }_{818}$ [3 5], but for now, ERP evidence remains elusive. 819

\section{Conclusions}

Using long-distance particle verb dependencies in German, we tested the $\quad 821$ hypothesis that uncertainty may affect readers' willingness to commit to a $\quad 822$ specific sentence meaning. Long-range lexical predictions may be advantageous 823 to interpreting an event in German particle verb dependencies where the full $\quad{ }_{824}$ 
verb form can be split across almost an entire sentence. However, we were 825 unable to find conclusive evidence that uncertainty affected lexical predictions, $\quad 826$ even in such a high-stakes context where predictions would have been $\quad 827$ particularly useful. This result could have been due to the comparison of ERPs 828 at implausible target words; however, if this was the case, it is surprising that $\quad{ }^{2} 2$ evidence against an effect of uncertainty — as measured by entropy — was so 830 inconclusive given clear predictions made by the literature about implausible $\quad 831$ input, and given that the study design was sufficient to demonstrate evidence $\quad 832$ for a standard cloze probability effect on the N400. 833

We did find evidence, however, that manipulating entropy was unlikely to $\quad 834$ produce changes in amplitude at large a priori hypothesised effect sizes. Thus, $\quad 835$ our findings do not rule out the possibility that an entropy effect might be $\quad 836$ present but small in magnitude. A small-magnitude entropy effect could suggest $\quad 837$ that the semantic violation caused by an implausible verb-particle combination 838 has less clear consequences for processing cost than the violations at multiple $\quad 839$ levels of representation caused by anomalous nouns in previous studies. This $\quad 840$ raises questions such as whether simpler violations are less costly or whether $\quad{ }_{841}$ they are more amenable to repair. The properties of particle verb dependencies $\quad 842$ therefore present an interesting way to extend our understanding of probabilistic $\quad 843$

$\begin{array}{ll}\text { processing cost. } & 844\end{array}$

\section{Supporting information $\quad 845$}

\section{S1 Appendix. Detail on pre-registrations 846}

Experiment 1 was pre-registered on OSF https://osf.io/qbna2. 847

Deviations from this pre-registration were: i) the number of critical trials $\quad 848$ analysed was 40 and not 44 as pre-registered (4 items were excluded for not $\quad 849$ meeting the criteria described under Materials); ii) we analysed a region of $\quad 850$ electrodes rather than the pre-registered single electrode, as region analyses are 851 
standard in the literature (see S2 Appendix for a comparison of the 852

single-electrode vs. region analysis results, in which our conclusions would have 853 been the same); iii) we used regularising priors with a standard deviation of $\quad 854$ $1 \mu V(\operatorname{Normal}(0,1))$ which assumed that ERP effects would vary with $95 \% \quad 855$ probability between $-2 \mu V$ of zero. In contrast, the pre-registered priors had 856 standard deviations of 5 and even $10 \mu \mathrm{V}$, which are less plausible effect sizes $\quad 857$ based on previous psycholinguistic ERP research. Moreover, regularising priors 858 better constrain the statistical model 67,70 ; iv) we added hypotheses and 859 analyses concerning the PNP whose existence we were unaware of at the time of 860 the pre-registration; v) we used Bayes factors to quantify evidence for/against $\quad 861$ effects rather than the pre-registered proportion of the posterior distribution $\quad{ }_{862}$ that was above zero, since the latter does not rule out a null effect or an effect 863 in the opposite direction if zero is a plausible value in the posterior [95]. 864

Experiment 2 was pre-registered at https://osf.io/y6k2d. The deviations 865 to the pre-registration were: i) the number of critical trials was 50 rather than $\quad 866$ 54 ; ii) we used regularising priors rather than the same priors pre-registered in $\quad 867$ Experiment 1; iii) we added Bayes factor analyses to inform inference. 868

\section{S2 Appendix. Pre-registered single electrode analyses for 869}

\section{$\begin{array}{ll}\text { Experiment 1. } & 870\end{array}$}

The dependent variables for Experiment 1 deviated from the pre-registration 871 where we proposed analysing a single electrode as a way of reducing averaging, $\quad 872$ which can artificially reduce variance [96]. However, the disadvantage of 873 analysing single electrodes is that ERP components are not so highly focal as to 874 appear at one electrode, and so the already high signal-to-noise ratio inherent in 875 ERP can drown out genuine effects or create the illusion of an effect where there 876 is none 97, 98. For this reason, later in Experiment 2, we pre-registered and $\quad 877$ analysed a region of electrodes based on the results of Experiment 1. To make 878 the presentation of results between both experiments consistent, we presented $\quad 879$ 


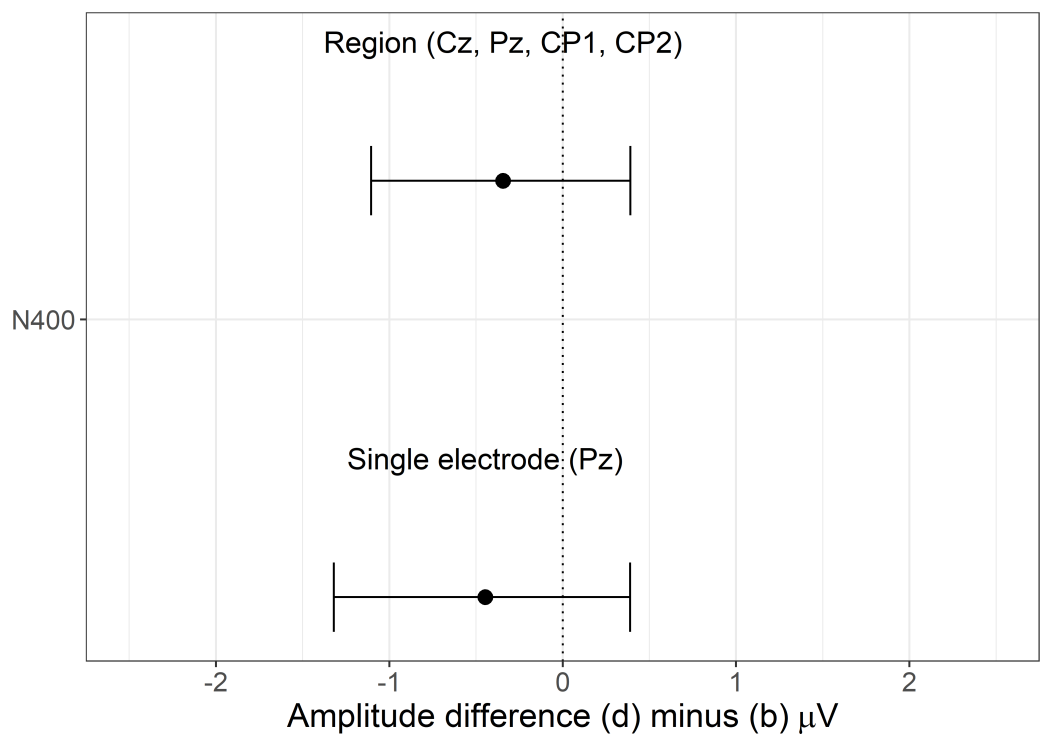

Fig 5. Comparison the results of the pre-registered single-electrode analysis of the $\mathrm{N} 400$ in Experiment 1 with the region-based analysis presented in the main manuscript. Points and errorbars represent the means and $95 \%$ credible intervals estimated for the comparison of 1-particle and $2+$ particle violations in the N400 time window. Both analysis approaches yield comparable estimates, confirming that the region analysis used in the main manuscript did not lead to more (or less) favourable conclusions about the N400.

region analyses for both experiments. However, for transparency, we present here 880 a comparison of the posterior estimates obtained via the pre-registered, single $\quad{ }_{881}$ electrode analysis of Experiment 1 with those obtained via the region analysis. $\quad{ }_{882}$

\section{Author contributions}

KS and TM developed the idea and design of the experiment. KS oversaw 884 design of the stimuli and data collection. KS analysed the data with input from 885 TM and SV. KS wrote the manuscript with input from TM and SV. 886

\section{Acknowledgments}

We thank Johanna Thieke, Romy Leue, Chiara Tschirner, and Alexandra $\quad 888$ Lorson for their help with designing the stimuli and collecting the data. 889 


\section{References}

1. Kuperberg G, Jaeger TF. What do we mean by prediction in language comprehension? Language Cognition \& Neuroscience. 2016;31(1). doi:10.1080/23273798.2015.1102299.

2. Pickering MJ, Gambi C. Predicting while comprehending language: A theory and review. Psychological Bulletin. 2018;144(10):1002-1044. doi:10.1037/bul0000158.

3. Husain S, Vasishth S, Srinivasan N. Strong expectations cancel locality effects: Evidence from Hindi. PloS one. 2014;9(7):e100986. doi:10.1371/journal.pone.0100986.

4. Safavi MS, Husain S, Vasishth S. Dependency resolution difficulty increases with distance in Persian separable complex predicates : Evidence against the expectation-based account. Frontiers in Psychology. 2016; p. 1-21. doi:10.3389/fpsyg.2016.00403.

5. Stone K, von der Malsburg T, Vasishth S. The effect of decay and lexical uncertainty on processing long-distance dependencies in reading. PeerJ. 2020;(8):e10438. doi:https://doi.org/10.7717/peerj.10438.

6. Rosenthal R. The file drawer problem and tolerance for null results. Psychological Bulletin. 1979;86(3):638-641. doi:10.1037/0033-2909.86.3.638.

7. Kutas M, Van Petten CK, Kluender R. Chapter 17 - Psycholinguistics Electrified II (1994-2005). In: Traxler MJ, Gernsbacher MA, editors. Handbook of Psycholinguistics (Second Edition). London: Academic Press; 2006. p. 659-724. Available from: https://doi.org/10.1016/B978-012369374-7/50018-3 
8. Pickering MJ, Barton S, Shillcock RC. Unbounded dependencies, island constraints and processing complexity. In: Clifton C, Frazier L, Rayner K, editors. Perspectives on sentence processing. London: Lawrence Erlbaum.; 1994. p. 199-224.

9. Ness T, Meltzer-Asscher A. When is the verb a potential gap site? The influence of filler maintenance on the active search for a gap. Language, Cognition and Neuroscience. 2019;34(7):936-948. doi:https://doi.org/10.1080/23273798.2019.1591471.

10. Wagers MW, Phillips C. Multiple dependencies and the role of the grammar in real-time comprehension1. Journal of Linguistics. 2009;45(2):395-433. doi:10.1017/S0022226709005726.

11. Phillips C, Wagers MW, Lau E. 5: Grammatical Illusions and Selective Fallibility in Real-Time Language Comprehension. Experiments at the Interfaces. 2011; p. 147-180. doi:10.1163/9781780523750-006.

12. Fiebach CJ, Schlesewsky M, Friederici AD. Separating syntactic memory costs and syntactic integration costs during parsing: the processing of German WH-questions. Journal of Memory and Language. 2002;47(2):250-272. doi:10.1016/S0749-596X(02)00004-9.

13. Lau E, Stroud C, Plesch S, Phillips C. The role of structural prediction in rapid syntactic analysis. Brain and Language. 2006;98(1):74-88. doi:10.1016/j.bandl.2006.02.003.

14. Phillips C, Kazanina N, Abada SH. ERP effects of the processing of syntactic long-distance dependencies. Cognitive Brain Research. 2005;22(3):407-428. doi:10.1016/j.cogbrainres.2004.09.012.

15. Staub A, Clifton C. Syntactic prediction in language comprehension: Evidence from either...or. Journal of Experimental Psychology: Learning, 
Memory, and Cognition. 2006;32(2):425-436.

doi:10.1037/0278-7393.32.2.425.

16. Frazier L, Clifton C. On Bound Variable Interpretations: The LF-Only Hypothesis. Journal of Psycholinguistic Research. 2000;29(2):125-140. doi:10.1023/A:1005136826534.

17. Ness T, Meltzer-Asscher A. Predictive Pre-updating and Working Memory Capacity: Evidence from Event-related Potentials. Journal of Cognitive Neuroscience. 2018;30(12):1916-1938.

doi:https://doi.org/10.1162/jocn_a_01322.

18. Lau E. Neural Indices of Structured Sentence Representation: State of the Art. In: Psychology of Learning and Motivation. vol. 68. Elsevier; 2018. p. 117-142. Available from: https: //linkinghub.elsevier.com/retrieve/pii/S0079742118300045

19. Staub A. The Effect of Lexical Predictability on Eye Movements in Reading: Critical Review and Theoretical Interpretation. Language and Linguistics Compass. 2015;9(8):311-327. doi:10.1111/lnc3.12151.

20. Hale J. Uncertainty About the Rest of the Sentence. Cognitive Science. 2006;30(4):643-672. doi:10.1207/s15516709cog0000_64.

21. Levy R. Expectation-based syntactic comprehension. Cognition. 2008;106(3):1126-1177. doi:10.1016/j.cognition.2007.05.006.

22. Smith NJ, Levy R. The effect of word predictability on reading time is logarithmic. Cognition. 2013;128(3). doi:10.1016/j.cognition.2013.02.013.

23. Frank SL, Otten LJ, Galli G, Vigliocco G. The ERP response to the amount of information conveyed by words in sentences. Brain and Language. 2015;140:1-11. doi:10.1016/j.bandl.2014.10.006. 
24. Lowder MW, Choi W, Ferreira F, Henderson JM. Lexical Predictability During Natural Reading: Effects of Surprisal and Entropy Reduction. Cognitive Science. 2018;doi:10.1111/cogs.12597.

25. Szewczyk J, Federmeier KD. Context-Based Facilitation of Semantic Access Follows Both Logarithmic and Linear Functions of Stimulus Probability. PsyArXiv. 2021;doi:10.31234/osf.io/rbych.

26. van Schijndel M, Linzen T. Single-Stage Prediction Models Do Not Explain the Magnitude of Syntactic Disambiguation Difficulty. Cognitive Science. 2021;45(6):e12988. doi:https://doi.org/10.1111/cogs.12988.

27. Michaelov J, Bergen B. How well does surprisal explain N400 amplitude under different experimental conditions? In: Proceedings of the 24th Conference on Computational Natural Language Learning. Online: Association for Computational Linguistics; 2020. p. 652-663. Available from: https://www. aclweb.org/anthology/2020.conll-1.53.

28. Elman JL, Hare M, McRae K. Cues, Constraints, and Competition in Sentence Processing. In: Beyond Nature-Nurture. Psychology Press; 2004.

29. McRae K, Spivey-Knowlton MJ, Tanenhaus MK. Modeling the Influence of Thematic Fit (and Other Constraints) in On-line Sentence Comprehension. Journal of Memory and Language. 1998;38(3):283-312. doi:10.1006/jmla.1997.2543.

30. Linzen T, Jaeger TF. Uncertainty and Expectation in Sentence Processing: Evidence From Subcategorization Distributions. Cognitive Science. 2016;40(6). doi:10.1111/cogs.12274.

31. Frank SL. Uncertainty Reduction as a Measure of Cognitive Load in Sentence Comprehension. Topics in Cognitive Science. 2013;5(3):475-494. doi:https://doi.org/10.1111/tops.12025. 
32. Federmeier KD, Wlotko EW, Ochoa-Dewald ED, Kutas M. Multiple effects of sentential constraint on word processing. Brain Research. 2007;(1146):75-84. doi:10.1016/j.brainres.2006.06.101.

33. Kuperberg G, Brothers T, Wlotko EW. A Tale of Two Positivities (and the N400): Distinct neural signatures are evoked by confirmed and violated predictions at different levels of representation. Journal of Cognitive Neuroscience. 2020;32(1):12-35. doi:https://doi.org/10.1162/jocn_a_01465.

34. Thornhill DE, Van Petten C. Lexical versus conceptual anticipation during sentence processing: Frontal positivity and N400 ERP components. International Journal of Psychophysiology. 2012;83(3):382-392. doi:10.1016/j.ijpsycho.2011.12.007.

35. Staub A, Grant M, Astheimer L, Cohen A. The influence of cloze probability and item constraint on cloze task response time. Journal of Memory and Language. 2015;82:1-17. doi:10.1016/j.jml.2015.02.004.

36. Brothers T, Wlotko EW, Warnke L, Kuperberg G. Going the Extra Mile: Effects of Discourse Context on Two Late Positivities During Language Comprehension. Neurobiology of Language. 2020;1(1):135-160. doi:https://doi.org/10.1162/nol_a_00006.

37. Kutas M. In the company of other words: Electrophysiological evidence for single-word and sentence context effects. Language and Cognitive Processes. 1993;8(4):533-572. doi:https://doi.org/10.1080/01690969308407587.

38. Federmeier KD, Kutas M. A rose by any other name: Long-term memory structure and sentence processing. Journal of memory and Language. 1999;41(4):469-495. doi:10.1006/jmla.1999.2660. 
39. Wlotko EW, Federmeier KD. Finding the right word: Hemispheric asymmetries in the use of sentence context information. Neuropsychologia. 2007;45(13):3001-3014. doi:10.1016/j.neuropsychologia.2007.05.013.

40. Paczynski M, Kuperberg G. Multiple influences of semantic memory on sentence processing: Distinct effects of semantic relatedness on violations of real-world event/state knowledge and animacy selection restrictions. Journal of Memory and Language. 2012;67(4):426-448. doi:10.1016/j.jml.2012.07.003.

41. Lai MK, Rommers J, Federmeier KD. The fate of the unexpected: Consequences of misprediction assessed using ERP repetition effects. Brain Research. 2021; p. 147290. doi:10.1016/j.brainres.2021.147290.

42. Szewczyk JM, Schriefers H. Prediction in language comprehension beyond specific words: An ERP study on sentence comprehension in Polish. Journal of Memory and Language. 2013;68(4):297-314. doi:https://doi.org/10.1016/j.jml.2012.12.002.

43. Kutas M, Federmeier KD. Thirty Years and Counting: Finding Meaning in the N400 Component of the Event-Related Brain Potential (ERP). Annual Review of Psychology. 2011;62(1):621-647. doi:10.1146/annurev.psych.093008.131123.

44. Van Petten C, Kutas M. Interactions between sentence context and word frequencyinevent-related brainpotentials. Memory \& Cognition. 1990;18(4):380-393. doi:https://doi.org/10.3758/BF03197127.

45. Payne BR, Lee CL, Federmeier KD. Revisiting the incremental effects of context on word processing: Evidence from single-word event-related brain potentials. Psychophysiology. 2015;52(11):1456-1469. doi:10.1111/psyp.12515. 
46. Van Petten C, Luka BJ. Prediction during language comprehension: Benefits, costs, and ERP components. International Journal of Psychophysiology. 2012;83(2):176-190. doi:http://dx.doi.org/10.1016/j.ijpsycho.2011.09.015.

47. Rabovsky M, Hansen SS, McClelland JL. Modelling the N400 brain potential as change in a probabilistic representation of meaning. Nature Human Behaviour. 2018;2(9):693. doi:10.1038/s41562-018-0406-4.

48. Kutas M, Hillyard SA. Brain potentials during reading reflect word expectancy and semantic association. Nature. 1984;307(5947):161-163. doi:https://doi.org/10.1038/307161a0.

49. Maess B, Mamashli F, Obleser J, Helle L, Friederici AD. Prediction Signatures in the Brain: Semantic Pre-Activation during Language Comprehension. Frontiers in Human Neuroscience. 2016;10. doi:10.3389/fnhum.2016.00591.

50. Fleur DS, Flecken M, Rommers J, Nieuwland MS. Definitely saw it coming? The dual nature of the pre-nominal prediction effect. Cognition. 2020;204:104335. doi:10.1016/j.cognition.2020.104335.

51. Hagoort P, Brown C, Groothusen J. The syntactic positive shift (sps) as an erp measure of syntactic processing. Language and Cognitive Processes. 1993;8(4):439-483. doi:https://doi.org/10.1080/01690969308407585.

52. Bornkessel-Schlesewsky I, Schlesewsky M. An alternative perspective on "semantic P600" effects in language comprehension. Brain Research Reviews. 2008;59(1):55-73. doi:10.1016/J.BRAINRESREV.2008.05.003.

53. Fitz H, Chang F. Language ERPs reflect learning through prediction error propagation. Cognitive Psychology. 2019;111:15-52. doi:10.1016/j.cogpsych.2019.03.002. 
54. Osterhout L, Holcomb PJ. Event-related brain potentials elicited by syntactic anomaly. Journal of Memory and Language. 1992;31(6):785-806. doi:10.1016/0749-596X(92)90039-Z.

55. Brouwer H, Crocker MW, Venhuizen NJ, Hoeks JCJ. A Neurocomputational Model of the N400 and the P600 in Language Processing. Cognitive Science. 2017;41(S6):1318-1352. doi:10.1111/cogs.12461.

56. Brouwer H, Hoeks JCJ. A time and place for language comprehension: mapping the N400 and the P600 to a minimal cortical network. Frontiers in Human Neuroscience. 2013;7. doi:10.3389/fnhum.2013.00758.

57. DeLong KA, Quante L, Kutas M. Predictability, plausibility, and two late ERP positivities during written sentence comprehension. Neuropsychologia. 2014;61(1). doi:https://doi.org/10.1016/j.neuropsychologia.2014.06.016.

58. Drummond A. Ibex: Software for psycholinguistic experiments; 2016. Available from: https://github.com/addrummond/ibex.

59. Mathôt S, Schreij D, Theeuwes J. OpenSesame: An open-source, graphical experiment builder for the social sciences. Behavior Research Methods. 2012;44(2):314-324. doi:10.3758/s13428-011-0168-7.

60. Jasper H. The 10/20 international electrode system. EEG and Clinical Neurophysiology. 1958;10(2):370-375.

61. Cardoso J. Blind signal separation: statistical principles. Proceedings of the IEEE. 1998;86(10):2009-2025. doi:10.1109/5.720250.

62. Makeig S, Bell AJ, Jung TP, Sejnowski TJ. Independent component analysis of electroencephalographic data. In: Advances in neural information processing systems; 1996. p. 145-151. 
63. Bell AJ, Sejnowski TJ. An Information-Maximization Approach to Blind Separation and Blind Deconvolution. Neural Computation. 1995;7(6):1129-1159. doi:https://doi.org/10.1162/neco.1995.7.6.1129.

64. Nicenboim B. eeguana: A package for manipulating EEG data in R; 2018. Available from: https://github.com/bnicenboim/eeguana.

65. Buerkner PC. brms: An R Package for Bayesian Multilevel Models Using Stan. Journal of Statistical Software. 2018;80(1). doi:10.18637/jss.v080.i01.

66. DeLong KA, Urbach TP, Kutas M. Probabilistic word pre-activation during language comprehension inferred from electrical brain activity. Nature neuroscience. 2005;8(8):1117. doi:https://doi.org/10.1038/nn1504.

67. Gelman A. Prior Choice Recommendations; 2020. Available from: https://github.com/stan-dev/stan.

68. Chung Y, Gelman A, Rabe-Hesketh S, Liu J, Dorie V. Weakly Informative Prior for Point Estimation of Covariance Matrices in Hierarchical Models. Journal of Educational and Behavioral Statistics. 2015;40(2):136-157. doi:https://doi.org/10.3102/1076998615570945.

69. Gelman A, Jakulin A, Pittau MG, Su YS. A weakly informative default prior distribution for logistic and other regression models. Annals of Applied Statistics. 2008;2(4):1360-1383. doi:10.1214/08-AOAS191.

70. Gelman A, Simpson D, Betancourt M. The Prior Can Often Only Be Understood in the Context of the Likelihood. Entropy. 2017;19(10):555. doi:10.3390/e19100555.

71. Jeffreys H. Theory of probability. Clarendon; 1961.

72. Lee M, Wagenmakers EJ. Bayesian Cognitive Modeling: A Practical Course. Cambridge: Cambridge University Press; 2013. 
73. Aitkin M. Posterior Bayes Factors. Journal of the Royal Statistical Society: Series B (Methodological). 1991;53(1):111-128. doi:10.1111/j.2517-6161.1991.tb01812.x.

74. Gelman A, Carlin J, Stern H, Dunson D, Vehtari A, Rubin B. Bayesian data analysis; 2013. Available from: https://doi.org/10.1201/b16018.

75. Lee M, Wagenmakers EJ. Bayesian Cognitive Modeling: A Practical Course. Cambridge: Cambridge University Press; 2014.

76. Schad DJ, Betancourt M, Vasishth S. Toward a principled Bayesian workflow: A tutorial for cognitive science. Psychological Methods. 2020;doi:https://doi.org/10.1037/met0000275.

77. Czypionka A, Golcher F, Błaszczak J, Eulitz C. When verbs have bugs: lexical and syntactic processing costs of split particle verbs in sentence comprehension. Language, Cognition and Neuroscience. 2019;34(3):326-350. doi:10.1080/23273798.2018.1539756.

78. Vasishth S, Yadav H, Schad D, Nicenboim B. Sample size determination for Bayesian hierarchical models commonly used in psycholinguistics. 2021;doi:10.31234/osf.io/u8yvc.

79. Szewczyk JM, Mech EN, Federmeier KD. Event-related potentials reveal rapid positive and negative updating of message-level representations. Potsdam; 2020.

80. Gelman A, Carlin J. Beyond Power Calculations: Assessing Type S (Sign) and Type M (Magnitude) Errors. Perspectives on Psychological Science. 2014;9(6):641-651. doi:https://doi.org/10.1177/1745691614551642.

81. Kim A, Osterhout L. The independence of combinatory semantic processing: Evidence from event-related potentials. Journal of Memory and Language. 2005;52(2):205-225. doi:10.1016/j.jml.2004.10.002. 
82. Kuperberg G, Sitnikova T, Caplan D, Holcomb PJ. Electrophysiological distinctions in processing conceptual relationships within simple sentences. Cognitive Brain Research. 2003;17(1):117-129.

doi:10.1016/S0926-6410(03)00086-7.

83. Meerendonk NVD, Kolk HHJ, Chwilla DJ, Vissers CTWM. Monitoring in Language Perception. Language and Linguistics Compass.

2009;3(5):1211-1224.

doi:https://doi.org/10.1111/j.1749-818X.2009.00163.x.

84. Metzner P, von der Malsburg T, Vasishth S, Rösler F. The Importance of Reading Naturally: Evidence From Combined Recordings of Eye Movements and Electric Brain Potentials. Cognitive Science. 2017;41. doi:10.1111/cogs.12384.

85. Gunter TC, Friederici AD, Schriefers H. Syntactic Gender and Semantic Expectancy: ERPs Reveal Early Autonomy and Late Interaction. Journal of Cognitive Neuroscience. 2000;12(4):556-568.

doi:https://doi.org/10.1162/089892900562336.

86. Hoeks JCJ, Stowe LA, Doedens G. Seeing words in context: the interaction of lexical and sentence level information during reading. Cognitive Brain Research. 2004;19(1):59-73. doi:10.1016/j.cogbrainres.2003.10.022.

87. van Herten M, Kolk HHJ, Chwilla DJ. An ERP study of P600 effects elicited by semantic anomalies. Cognitive Brain Research. 2005;22(2):241-255. doi:10.1016/j.cogbrainres.2004.09.002.

88. Osterhout L, Mobley LA. Event-Related Brain Potentials Elicited by Failure to Agree. Journal of Memory and Language. 1995;34(6):739-773. doi:10.1006/jmla.1995.1033. 
89. Friederici AD. The Time Course of Syntactic Activation During Language Processing: A Model Based on Neuropsychological and Neurophysiological Data. Brain and Language. 1995;50(3):259-281. doi:10.1006/brln.1995.1048.

90. Vissers CTWM, Chwilla DJ, Kolk HHJ. Monitoring in language perception: The effect of misspellings of words in highly constrained sentences. Brain Research. 2006;1106(1):150-163. doi:10.1016/j.brainres.2006.05.012.

91. Piai V, Meyer L, Schreuder R, Bastiaansen MCM. Sit down and read on: Working memory and long-term memory in particle-verb processing. Brain and Language. 2013;127(2):296-306.

doi:10.1016/j.bandl.2013.09.015.

92. Brown C, Hagoort P. The Processing Nature of the N400: Evidence from Masked Priming. Journal of Cognitive Neuroscience. 1993;5(1):34-44. doi:10.1162/jocn.1993.5.1.34.

93. Van Berkum J, Hagoort P, Brown C. Semantic Integration in Sentences and Discourse: Evidence from the N400. Journal of Cognitive Neuroscience. 1999;11(6):657-671. doi:https://doi.org/10.1162/089892999563724.

94. Hagoort P. Reflections on the Neurobiology of Syntax; 2009.

95. Wagenmakers EJ, Lee MD, Rouder JN, Morey RD. The Principle of Predictive Irrelevance, or Why Intervals Should Not be Used for Model Comparison Featuring a Point Null Hypothesis. Annals of Theoretical Psychology book series. 2020;16:20.

96. Barr DJ, Levy R, Scheepers C, Tily HJ. Random effects structure for confirmatory hypothesis testing: Keep it maximal. Journal of Memory and Language. 2013;68(3):255-278. doi:10.1016/j.jml.2012.11.001. 
97. Luck SJ. Ten Simple Rules for Designing and Interpreting ERP

Experiments. In: Handy TC, editor. Event-related Potentials: A Methods Handbook. MIT press; 2005. p. 17-32.

98. Luck SJ, Gaspelin N. How to Get Statistically Significant Effects in Any ERP Experiment (and Why You Shouldn’t). Psychophysiology. 2016;4424. doi:10.1111/psyp.12639. 